\title{
Low-Pass Filter Properties of Basal Ganglia-Cortical-Muscle Loops in the Normal and MPTP Primate Model of Parkinsonism
}

\author{
Michal Rivlin-Etzion,,${ }^{1,2}$ Odeya Marmor, ${ }^{1}$ Guy Saban, ${ }^{1}$ Boris Rosin, ${ }^{1}$ Suzanne N. Haber, ${ }^{4}$ Eilon Vaadia, ${ }^{1,2}$ Yifat Prut,${ }^{1,2}$ \\ and Hagai Bergman ${ }^{1,2,3}$ \\ ${ }^{1}$ Department of Physiology, The Hebrew University-Hadassah Medical School, Jerusalem 91120, Israel, ${ }^{2}$ The Interdisciplinary Center for Neural \\ Computation and ${ }^{3}$ Eric Roland Center for Neurodegenerative Diseases, The Hebrew University, Jerusalem 91904, Israel, and ${ }^{4}$ Department of Pharmacology \\ and Physiology, University of Rochester, Rochester, New York 14642
}

Oscillatory bursting activity is commonly found in the basal ganglia (BG) and the thalamus of the parkinsonian brain. The frequency of these oscillations is often similar to or higher than that of the parkinsonian tremor, but their relationship to the tremor and other parkinsonian symptoms is still under debate. We studied the frequency dependency of information transmission in the cortex-BG and cortex-periphery loops by recording simultaneously from multiple electrodes located in the arm-related primary motor cortex (MI) and in the globus pallidus (GP) of two vervet monkeys before and after 1-methyl-4-phenyl-1,2,3,6-tetrahydropyridine (MPTP) treatment and induction of parkinsonian symptoms. We mimicked the parkinsonian bursting oscillations by stimulating with $35 \mathrm{~ms}$ bursts given at different frequencies through microelectrodes located in MI or GP while recording the evoked neuronal and motor responses. In the normal state, microstimulation of MI or GP does not modulate the discharge rate in the other structure. However, the functionalconnectivity between MI and GP is greatly enhanced after MPTP treatment. In the frequency domain, GP neurons usually responded equally to $1-15 \mathrm{~Hz}$ stimulation bursts in both states. In contrast, MI neurons demonstrated low-pass filter properties, with a cutoff frequency above $5 \mathrm{~Hz}$ for the MI stimulations, and below $5 \mathrm{~Hz}$ for the GP stimulations. Finally, muscle activation evoked by MI microstimulation was markedly attenuated at frequencies higher than $5 \mathrm{~Hz}$. The low-pass properties of the pathways connecting GP to MI to muscles suggest that parkinsonian tremor is not directly driven by the BG $5-10 \mathrm{~Hz}$ burst oscillations despite their similar frequencies.

Key words: primate; microstimulation; globus pallidus; motor cortex; frequency domain; transfer function; Parkinson's disease

\section{Introduction}

The BG are commonly viewed as operating in a feedforward mode. They receive projections from all cortical areas and feed this information forward via the thalamus to the frontal cortex or directly to brainstem motor nuclei (Shink et al., 1997; MenaSegovia et al., 2004; McHaffie et al., 2005). The cortical and brainstem motor centers in turn transmit this information down to the spinal cord and muscles (Albin et al., 1989). The functionalconnectivity between the different components of this system has often been studied using electrical stimulation. Such an approach was used to map the relations between MI and muscles (Asanuma and Rosen, 1972; Palmer and Fetz, 1985) MI and BG (Ryan and Clark, 1991; Maurice et al., 1999; Nambu et al., 2000), as well as GP and muscles (Horak and Anderson, 1984).

This simplified view of the BG provided profound insights

Received July 26, 2007; revised 0ct. 7, 2007; accepted 0ct. 30, 2007.

This work was partly supported by a Hebrew University Netherlands Association grant entitled "Fighting against Parkinson" (H.B.) and by a graduate student fellowship from the Lily Safra Interdisciplinary Center for Neural Computation (M.R.-E.).

Correspondence should be addressed to Michal Rivlin-Etzion, Department of Physiology, The Hebrew UniversityHadassah Medical School, P.0. Box 12272, Jerusalem 91120, Israel. E-mail: michal.rivlin@mail.huji.ac.il. DOI:10.1523/JNEUROSCI.3388-07.2008

Copyright $\odot 2008$ Society for Neuroscience $\quad 0270-6474 / 08 / 280633-17 \$ 15.00 / 0$ into the pathophysiology of BG related disorders (DeLong, 1990), and led to the development of the current neurosurgical treatment of Parkinson's disease (PD) (Bergman et al., 1990; Benabid et al., 2006). However, the BG are more than a simple feedforward network, because of their reciprocal closed-loop structure (Alexander et al., 1986; Joel and Weiner, 1994; Leblois et al., 2006; Rivlin-Etzion et al., 2006). The frontal cortex, the target of BG output, is one of the main cortical areas projecting to the striatum. Moreover, the cortical projections to the brainstem and spinal cord are paralleled by ascending afferent pathways, closing the loop between the frontal cortex and the periphery.

In principle, electrical stimulation could also be used to study information transfer in closed-loop neural network, although the interpretation of the obtained response might be confounded by the fact that information may be transmitted orthodromically and antidromically (i.e., from the soma to the axon terminals and vice versa). In this study, we explored the functional connectivity within the GP-cortex-muscle loops by stimulating through microelectrodes inserted into either MI or GP, while recording the evoked activity in both structures as well as contralateral arm movements. Because the GP and MI are indirectly connected (the striatum and the subthalamic nucleus are positioned along the pathway from the cortex to the GP, and the thalamus is similarly 
located between the GP and MI), microstimulation effects are probably mediated mainly through orthodromic conduction.

Most connectivity studies have used a single pulse or a brief train of electrical stimulation (Asanuma and Rosen, 1972; Cheney and Fetz, 1985; Tremblay et al., 1989; Kita, 1992; Nambu et al., 2000), although an alternative approach was presented in which a prolonged pattern of electrical stimulation was applied (Graziano et al., 2002). Unlike previous studies, we used a stimulus pattern that contained $35 \mathrm{~ms}$ bursts delivered at different frequencies ranging from 1 to $15 \mathrm{~Hz}$, in an attempt to mimic the oscillatory bursting pattern often encountered in the BG of parkinsonian patients (Weinberger et al., 2006) and MPTP-treated primates (Nini et al., 1995; Heimer et al., 2006). This pattern can be used to characterize the system transfer functions in the frequency domain (Lathi, 2005) and thus reveal the spectral properties of the BG-cortex-muscle network.

\section{Materials and Methods}

Animals. Two vervet (African green, Cercopithecus aethiops) monkeys (T and $\mathrm{W}$; females; weighing 3 and $3.5 \mathrm{~kg}$, respectively) were used in this study. Before any of the other procedures were performed, the monkeys were trained to sit in a primate chair, to permit handling by the experimenter, and became familiarized with the laboratory setting. The monkeys had access to standard primate chow and water ad libitum during the whole experimental period. The monkeys' health was monitored by a veterinarian, and their weights and clinical status were checked daily. All experimental protocols were performed in accordance with the National Institutes of Health Guide for the Care and Use of Laboratory Animals and the Hebrew University guidelines for the use and care of laboratory animals in research and were approved and supervised by the Institutional Animal Care and Use Committee.

Surgical procedures. We attached an MRI-compatible plastic (Cilux) head holder and a $27 \mathrm{~mm}^{2}$ recording chamber to the monkey's skull to allow access to the arm-related areas of the primary motor cortex and to the GP of the right hemisphere (Fig. $1 A, B$ ). The recording chamber was tilted $40^{\circ}$ laterally in the coronal plane, with its center targeted at the following stereotaxic coordinates (in $\mathrm{mm}$ ): monkey $\mathrm{T}$, anterior 12, lateral 7 , height 12 (above interaural line); monkey $\mathrm{W}$, anterior 10, lateral 5, height 14 (Contreras et al., 1981; Martin and Bowden, 2000), and webbased digital brain atlas of the vervet monkey, currently at http://labs.pharmacology.ucla.edu/mellab/vervet_atlas. The chamber coordinates were verified using magnetic resonance imaging (MRI). The MRI scan [GE Healthcare (Little Chalfont, UK) 1.5 Tesla system; fast spin echo sequence; $\mathrm{TR}=5.4 \mathrm{~s} ; \mathrm{TE}=46.49 \mathrm{~ms}$; Echo train length $=12$; number of averages $=4 ; \mathrm{FOV}=12 \times 12 \mathrm{~cm} ; 256 \times 256$ pixels; coronal slices $2 \mathrm{~mm}$ wide] was performed with tungsten electrodes at different $\mathrm{X}-\mathrm{Y}$ coordinates of the chamber (Fig. $1 \mathrm{~A}$ ). We then aligned the two-dimensional MRI images with coronal sections of the primate atlas.

Surgical procedures were performed under deep isoflurane and $\mathrm{N}_{2} \mathrm{O}$ inhalation general anesthesia. Analgesia and antibiotics were administered during surgery and continued for $2 \mathrm{~d}$ postoperatively. MRI procedures were performed under light IM Dormitor and Ketamine anesthesia. Recording began after a postoperative recovery period of $6 \mathrm{~d}$ in both monkeys.

Recording and structure identification. During recording sessions, the animals were awake and seated in a primate chair with their head and right hand restrained, but free to move their trunk and their left arm (contralateral to the recording hemisphere) and legs. Four glass-coated $150 \mu \mathrm{m}$ shaft diameter tungsten microelectrodes (impedance, $0.2-0.5$ $\mathrm{M} \Omega$ at $1 \mathrm{kHz})$, confined within a cylindrical metal guide $(1.36 / 1.65 \mathrm{~mm}$ inner/outer diameter) were advanced separately (EPS; Alpha Omega Engineering, Nazareth, Israel) into the arm-related area of the motor cortex. A similar set of four independently controlled microelectrodes targeted the pallidum through the same chamber (Double MT; Alpha Omega Engineering) (Fig. $1 \mathrm{~B}$ ). Each electrode signal was amplified with a gain of 5000 and bandpass filtered with a $300-6000 \mathrm{~Hz}$ (monkey T) and a 1-6000 Hz (monkey W) four-pole Butterworth filter (MIP+; Alpha-
Omega Engineering). The signal was continuously sampled at $25 \mathrm{kHz}$ with 12-bits $\pm 5 \mathrm{~V}$ A/D converter (Alpha-Map; Alpha Omega Engineering). During the acquisition of the neuronal data, two experimenters controlled the position and spike sorting of the eight electrodes. Spikes were detected from the filtered analog data $(300-6000 \mathrm{~Hz}$ for both monkeys) by on-line detection and sorting software using a templatematching algorithm (MSD; Alpha Omega Engineering). The quality of the detection and spike sorting was estimated and graded on-line every 3 min by the experimenters. This on-line quality estimation was based on three criteria: (1) the superimposed analog traces of the recently (20$100)$ sorted spikes and the waveforms of events that crossed an amplitude threshold that was set by the experimenter, (2) the cumulative distribution of the Euclidean distances between the detected events and the detection template (ASD; Alpha Omega Engineering), and (3) the stability of the discharge rate. The sampling rate of spike detection pulses was 28.57 and $40 \mathrm{kHz}$ for monkey $\mathrm{T}$ and $\mathrm{W}$, respectively.

Mapping of the motor cortex was done by examination of the neural responses to passive movements of the contralateral limbs and by electrical stimulations through the microelectrodes using an optically coupled isolator and linear current-source stimulator (Alpha Omega Engineering). The stimulation pattern used for the mapping consisted of 50 ms of a $300 \mathrm{~Hz}$ burst ( 15 pulses) of biphasic symmetric pulses, each phase $0.2 \mathrm{~ms}$, with negative pulse leading. Stimulation current amplitude during mapping ranged from 5 to $80 \mu \mathrm{A}$. The boundaries of the arm related area of the primary motor cortex were determined as covering the area in which stimulation of $15 \mu \mathrm{A}$ or less caused a movement of the forearm, wrist or fingers.

Entry into the pallidum (the lateral border of the GPe) was easily recognized in our penetrations because of the considerably different firing rate, pattern, and spike shape of pallidal versus striatal neurons. The classification of each recorded cell as belonging to either the external or internal pallidum was determined as follows: Neurons located at a depth of $<1.5 \mathrm{~mm}$ from the striatopallidal border, or neurons that exhibited pauses in their firing pattern were categorized as GPe neurons (DeLong, 1971; Elias et al., 2007). Candidates to GPi were neurons with a highfrequency discharge rate with no pauses, $2.5 \mathrm{~mm}$ from the striatopallidal border or farther in the ventromedial direction. Final classification as GPi was based on other physiological identifications along the electrode trajectory (e.g., border cells), or firing pattern of the cell (which was considered only in the normal state). If the classification as GPe or GPi was in doubt, the unit was excluded from the analysis. In this study we did not focus on recording from the GP hand- or motor-related area, but rather attempted to cover most of the areas of both GP segments.

Stimulation pattern. The stimulation pattern used for this study consisted of bursts of current pulses. Each burst contained eight biphasic symmetric pulses (each phase $0.2 \mathrm{~ms}$, with negative pulse leading) given at $200 \mathrm{~Hz}$, leading to bursts of $35 \mathrm{~ms}$ in length. The stimulation bursts were administered at different frequencies: $1,2,5$ and $10 \mathrm{~Hz}$, and in addition, $15 \mathrm{~Hz}$ for monkey $\mathrm{W}$ only. The stimulation in each of the frequency tests lasted $20 \mathrm{~s}$ (resulting, for example, in 20 bursts for the 1 $\mathrm{Hz}$ stimulation and 300 bursts for the $15 \mathrm{~Hz}$ stimulation) (Fig. 1C), with an interstimulus interval (IStimI) of $15 \mathrm{~s}$ before the first frequency test, between the different frequency tests, and after the last one. The order of frequency tests was determined randomly for each of the monkeys but remained constant throughout the experiment (monkey T: 5, 10, 1, $2 \mathrm{~Hz}$, monkey W: 10, 1, 2, 5, $15 \mathrm{~Hz}$ ).

After situating the electrodes in positions that enabled optimal recording of single units, the stimulation pattern was given through one or more electrodes (usually one, $89.2 \%$ of all cases), whereas all other electrodes were used for recording. All electrodes used in this study were of the same type (glass coated tungsten microelectrode) and could be used for either stimulation or recording. The electrodes used for stimulation within a certain session were connected to the current source, and were not used for recording during the same session. However, the same electrode could serve for stimulation in one session and for recording in the next session. The switch between stimulation and recording mode was controlled electronically (MIP; Alpha Omega Engineering). The amplification gain of the recording electrodes was reduced from 5000 to 5 during the stimulation pulses ( $0.3 \mathrm{~ms}$ before until $0.1 \mathrm{~ms}$ after the pulse) 
A

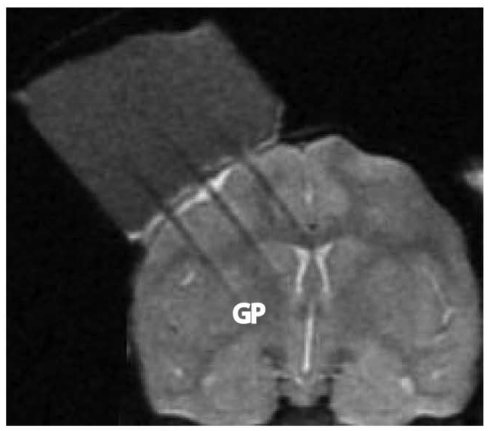

B
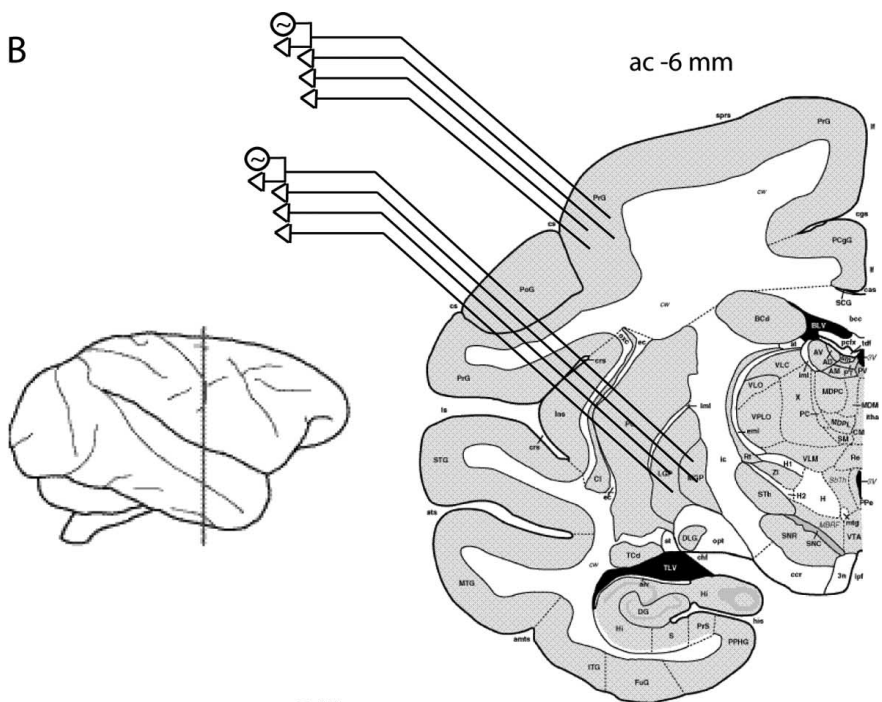

ac $-6 \mathrm{~mm}$

C
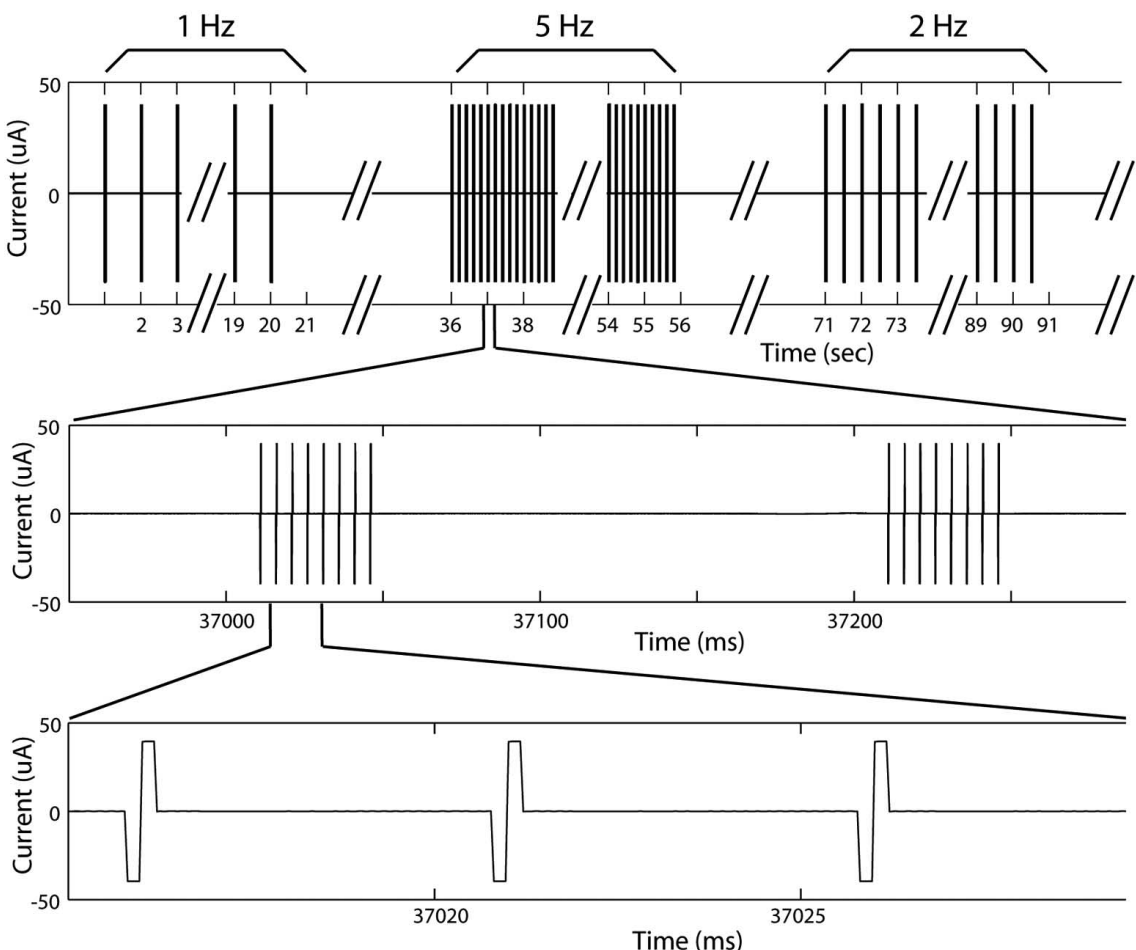

D
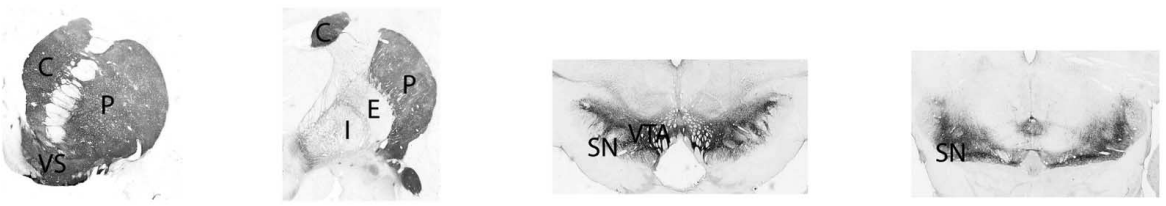

$\mathrm{E}$
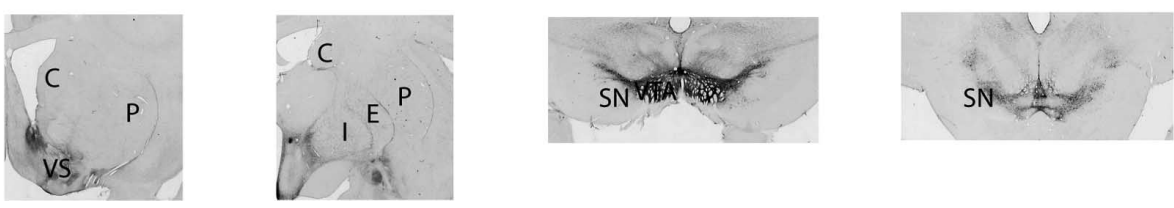

Figure 1. Experimental procedure and histology. $A, M R I$ of monkey W. An example of a coronal image. Three tungsten electrodes separated by a horizontal distance of $6 \mathrm{~mm}$ were inserted in the same coronal plane (ac $-2,2 \mathrm{~mm}$ posterior to the anterior commissure) through the recording chamber. The recording chamber is filled with $3 \%$ agar. B, Left, A schematic illustration showing the location of ac -6 coronal plane. The coronal plane crosses the central sulcus and the primary motor cortex. Right, A scheme of ac -6 coronal plane and the experimental setup: four electrodes in the GP and four electrodes in the motor cortex. Any electrode could be used as a stimulating electrode, while the other electrodes are recording. The atlas scheme is (Figure legend continues.) 
to reduce the saturation of the recording devices and to minimize the stimulus artifact. In this study, we include only stimulating sessions in which all stimulating electrodes were located in the same structure - MI or GP. Because of possible current leakage from the GPi to GPe (or vice-versa) that can occur when stimulating at the borders of these nuclei (Ranck, 1975), we did not attempt to distinguish between stimulating sessions in the GPe and GPi. For each stimulating electrode we used current amplitudes of $40 \mu \mathrm{A}$ in monkey $\mathrm{T}$, and either 40 or $60 \mu \mathrm{A}$ in monkey $\mathrm{W}(40 \mu \mathrm{A}$ were given in $33 \%$ and $55 \%$ of the sessions in the normal and MPTP states, respectively). When stimulating through more than one electrode, all the stimulating electrodes were connected to the same current source and the amplitude of the current was multiplied by the number of stimulating electrodes. We assumed an equal current distribution through the electrodes because of their similar impedance. The percentage of sessions with a single stimulating electrode in each of the structures (MI and GP) was similar in the normal and MPTP states (89\%), to avoid bias between the states. Nonetheless, we did not observe qualitative changes after stimulation with different numbers of electrodes, and therefore all stimulation sessions were pooled.

Data analysis. Cells were selected for recording as a function of their signal-to-noise ratio and real-time assessment of their isolation quality. Only stable (off-line verification of the stability of the neurons' firing rates throughout the stimulation session; we discarded any neuron that demonstrated a trend of decaying or increasing firing rate because this is indicative of possible neuronal injuries or unstable electrode position) and well isolated (as judged by the experimenters during real time) units were included in the analysis database of this study. Because of stimulus artifacts (see below), off-line quantification of the isolation quality (Joshua et al., 2007) could not be applied to the data set.

The stimuli produced prominent artifacts in the recordings, including short-term saturations of the recording electrodes. The nonsaturating artifacts could be overcome by stimulus artifact removal techniques (Wichmann, 2000; Hashimoto et al., 2002; Bar-Gad et al., 2004). However, in this study we chose to delete all stimulation periods from the neural data, including a $5 \mathrm{~ms}$ epoch after the last pulse, to provide a sufficient interval for the fading of the stimulus artifact. We therefore excluded a period of $40 \mathrm{~ms}$ for each stimulation burst from the data analysis.

We classified the neurons into different populations according to their location and the location of the stimulating electrodes. Thus, we studied the responses of MI neurons to GP stimulation ( $\mathrm{GP} \rightarrow \mathrm{MI}$ ), responses of MI neurons to MI stimulation $(\mathrm{MI} \rightarrow \mathrm{MI})$, as well as $\mathrm{MI} \rightarrow \mathrm{GPe}$, $\mathrm{MI} \rightarrow \mathrm{GPi}, \mathrm{GP} \rightarrow \mathrm{GPe}$ and $\mathrm{GP} \rightarrow \mathrm{GPi}$. Each population was further divided into the normal and MPTP states. We applied two main analyses on each neural population: peristimulus time histogram (PSTH) analysis and "decrease-increase" analysis, as detailed below.

Averaged population response: PSTH analysis. Responses to stimulation bursts of each neuron $(n)$ were first characterized in each frequency stimulation $(f)$ by their PSTH, constructed in $5 \mathrm{~ms}$ bins for a period of $600 \mathrm{~ms}$ around the stimulus (see examples in Figs. 2, 3):

\footnotetext{
(Figure legend continued.) adapted from Martin and Bowden (2000). C, The stimulation pattern includes $35 \mathrm{~ms}$ bursts with intraburst frequency of $200 \mathrm{~Hz}$ (8 pulses per burst); each pulse is symmetric biphasic, negative phase leading. The bursts were given at different frequencies for $20 \mathrm{~s}: 1$ (a total of 20 bursts), $2,5,10 \mathrm{~Hz}$, and, for monkey W, also $15 \mathrm{~Hz}$ (a total of 300 bursts). The interval between the frequency tests was $15 \mathrm{~s}$, and the order of frequencies was $5,10,1,2$ and, $10,1,2,5,15 \mathrm{~Hz}$, for monkeys T and W, respectively. $\boldsymbol{D}, \boldsymbol{E}$, Photomicrographs of TH staining demonstrating the loss of dopaminergic substantia nigra pars compacta neurons in the MPTPtreated monkey $T$ compared with a control animal. D was taken from a control normal macaque monkey; $\boldsymbol{E}$ is from the MPTP-treated monkey T (vervet). The photomicrographs illustrate the levels of rostral striatum (column 1), central striatum (column 2), and midbrain (columns 3, 4). Note the lack of TH-positive staining throughout the striatum with the exception of the ventral striatum, particularly the shell region. TH-positive cells are selectively lost in the ventral tier but spared in the ventral tegmental area. C, Caudate; $\mathrm{P}$, putamen; VS, ventral striatum; SN, substantia nigra; VTA, ventral tegmental area; E, GPe; I, GPi.
}

$$
\text { response } \operatorname{PSTH}_{n, f}=\frac{\sum_{i=1}^{i_{f}} \operatorname{Stim}_{\operatorname{trial}}(i)}{i_{f}},
$$

where Stim $\operatorname{trial}_{n, f}(i)$ is the response of neuron $n$ to the $i$ th burst given in frequency test $f$ (includes $100 \mathrm{~ms}$ before burst and $500 \mathrm{~ms}$ after beginning of the burst), and $i_{f}$ is the total number of bursts in frequency test $f$. When illustrating the response PSTH of single neurons (see Figs. 2, 3) we further smoothed the PSTH with a Gaussian window, $\sigma=15 \mathrm{~ms}$.

The PSTH of each neuron was then normalized by its spontaneous firing rate, which was determined by the mean firing rate in the last $10 \mathrm{~s}$ of all IStimIs (a total of 50 and $60 \mathrm{~s}$ in monkeys $\mathrm{T}$ and $\mathrm{W}$, respectively). Normalization was used to avoid bias of population means attributable to neurons with a high discharge rate, and was essential especially in the MI population, in which many of the neurons had a low discharge rate (see Table 3), and outlier neurons with a high discharge rate could bias the population behavior.

To estimate the population response we averaged the normalized PSTH for each frequency independently over all cells and obtained the averaged normalized response. Repeating the analysis with the nonnormalized discharge rates led to qualitatively similar results. In the average PSTH analysis, as well as in all other analyses, neurons from the two monkeys usually demonstrated similar behavior. Therefore, unless specified otherwise, neurons from monkeys $\mathrm{T}$ and $\mathrm{W}$ were pooled.

Modulations in firing rate: decrease-increase analysis. The average PSTH characterizes the population response only if the responses of the neurons are not divergent (i.e., most or all neurons in the population tend to increase their firing rate together, or decrease the rate together). In any other case, opposing modulations may cancel each other in the average PSTH, and thus will be "lost" to the analysis. Therefore, in addition to the PSTH analysis, we separated the responses of each neuron into bins with increases and decreases in firing rate. Significant modulations in the firing rate of a single neuron were identified by comparing each bin in the response PSTH of the neuron with its matched IStimI mean discharge rate. To do so, we calculated the "IStimI PSTH" of each neuron $(n)$ for each frequency $(f)$ based on the IStimI epochs:

$$
\operatorname{IStimI~PSTH}_{n, f}=\frac{\sum_{i=1}^{i_{f}}{\operatorname{IStimI~} \operatorname{trial}_{n, f}(i)}_{i_{f}}}{,}
$$

where IStimI $\operatorname{trial}_{n, f}(i)$ is a "trial" of $600 \mathrm{~ms}$ chosen randomly from all IStimI epochs of neuron $n$, and $i_{f}$ is the total number of bursts in frequency test $f$. We then calculated the mean and the SD of the "IStimI PSTH" and compared the values of the response PSTH with these expected values. Bins in which the response of the neuron deviated from the mean of the IStimI PSTH by $>2.58$ SD $(p<0.01)$ were considered to have significant increases or decreases. Based on this single neuron analysis, we determined the fraction of neurons in the population that significantly increased or significantly decreased their firing rate in every bin. Finally, for each population, frequency, and state, we calculated the maximum fraction of neurons that responded by increasing (decreasing) their discharge rate according to the bin with the maximum response percentage. This bin also determined the mean latency of the response (we chose not to define the latency according to response onset time, because the response could start before the stimulation burst ended). Response duration was calculated as the largest continuous period in which at least $50 \%$ of the responding neurons had a significant $(p<$ 0.01 ) change in their firing rate.

In the decrease-increase analysis we used different bin sizes for MI and GP neurons, as well as for different stimulation frequencies. For GP neurons, which had a high discharge rate (see Table 3) we used $10 \mathrm{~ms}$ bins, and for the MI neurons that fired at a much lower rate we used 25 ms bins for the 1, 2 and $5 \mathrm{~Hz}$ stimulations. Because the power of the test was higher in the high stimulation frequencies that contained many repetitions of stimulation bursts, we used $10 \mathrm{~ms}$ bins for the $10 \mathrm{~Hz}$ frequency for MI neurons as well. In the $15 \mathrm{~Hz}$ stimulation frequency (monkey W), 
the time lapse between the bursts was very short $(\sim 66.7 \mathrm{~ms}$, which left $\sim 26.7 \mathrm{~ms}$ after excluding the $40 \mathrm{~ms}$ stimulation and artifact period) and therefore the $15 \mathrm{~Hz}$ stimulation tests were not included in the decreaseincrease analysis.

Diversity index. Although the decrease-increase analysis reveals the prevalence of coincident modulations in the firing rate, it is not always easy to interpret. To simplify the interpretation, we defined a "diversity index" for each population:

$$
\operatorname{diversity}_{\mathrm{pop}, f}=1-\underset{i}{\operatorname{mean}}\left|\frac{I_{\mathrm{pop}, f}(i)-D_{\mathrm{pop}, f}(f)}{I_{\mathrm{pop}, f}(i)+D_{\mathrm{pop}, f}(i)}\right| \text {, }
$$

where $|\cdot|$ represents the absolute value, $I_{\text {pop }, f}(i)$ and $D_{\text {pop, } f}(i)$ are the percentages of neurons that significantly increased and decreased, respectively, their firing rates in the $i$ th bin in frequency test $(f)$ and population (pop). Bins included in the calculation of the diversity index start from the end of the first stimulation burst (time $40 \mathrm{~ms}$ ) and end at time $500 \mathrm{~ms}$ or before the beginning of the next stimulation burst (in the high frequencies). The index ranges between 0 and 1 . If $I=D$ (and both greater than 0 ) over all bins the index equals 1 , that is to say that the population did not tend to increases or decreases alone, but rather exhibited both kinds of modulations simultaneously and therefore was highly divergent. If, for example, $I>0$ and $D=0$, the index value is 0 ; in other words, the population did not show opposing modulations simultaneously, and therefore was not divergent. If both $I=0$ and $D=0$ for all bins (here we defined zero as $I, D<0.01$ although taking a smaller value produced similar results), the population responded neither by increases nor decreases to the stimulation test, and therefore the diversity index was set to 0 for this test.

Accelerometers. We used a uniaxial accelerometer (ACC) (8630C5; Kistler, Amherst, NY) to assess hand movements. Both monkeys had the accelerometer fastened to the back of their nonrestrained left wrist (contralateral to the stimulating hemisphere). The accelerometer output was amplified with a gain ranging from 4 to 20 (depending on the amplitude of the movement caused by the cortical stimulation) and bandpass filtered with a 1-250 Hz four-pole Butterworth filter (MIP+; Alpha Omega Engineering). The analog output of the accelerometers was sampled at 12500 and $781 \mathrm{~Hz}$ in monkeys $\mathrm{T}$ and $\mathrm{W}$, respectively. In the population analysis, the data were further digitally filtered with an eighth order Chebyshev type I $20 \mathrm{~Hz}$ low-pass filter and re-sampled at $200 \mathrm{~Hz}$ for both monkeys. Spontaneous movements, as well as other artifacts were noticeable in the accelerometer data from time to time. We therefore only included accelerometer records that had a good signal-to-noise ratio and did not include such spontaneous movements or artifacts. Because stimulation caused a different profile of movement in different recording sessions, the average population response of the arm acceleration was calculated from the normalized absolute value of the accelerometer output. Normalization of the acceleration value was obtained as for the neural data. In each recording session, an "IStimI PSTH" was derived for each of the frequencies based on the absolute ACC value in the IStimI periods, with a number of segments equal to the number of bursts delivered at that frequency. Then, each response PSTH of the absolute ACC output was normalized by its corresponding baseline IStimI mean value.

MPTP treatment and perfusion. After a period of recording in the normal state, Parkinsonism was induced by five intramuscular injections of $0.4 \mathrm{mg} / \mathrm{kg}$ 1-methyl-4-phenyl-1,2,3,6-tetrahydropyridine-HCl (MPTP; Sigma, Rehovot, Israel). The MPTP injections were given under light intramuscular ketamine hydrochloride $(10 \mathrm{mg} / \mathrm{kg})$ anesthesia and over a period of $4 \mathrm{~d}$ ( 3 injections in the first $24 \mathrm{~h}$ ). The clinical state of the monkeys was assessed daily according to a primate scale of Parkinsonism (Benazzouz et al., 1995). During termination of the recording days in the MPTP state we treated monkey T with $0.5 \times 25 / 250 \mathrm{mg}$ of Dopicar (L3,4-dihydroxyphenylalanine and carbidopa; Teva Pharmaceutical Industries, Petach Tikva, Israel) twice per day to verify the diagnosis of Parkinsonism by significant clinical improvement achieved with dopamine-replacement treatment. The drugs were administered orally as crushed powder dissolved in liquid. At the end of the experiment, monkey $\mathrm{T}$ was deeply anesthetized with a lethal dose of pentobarbital and perfused through the heart with saline, followed by a $4 \%$ paraformaldehyde fixative solution. Monkey W was perfused in a similar way within 30
Table 1. Summary of the neuronal database

\begin{tabular}{cllll}
\hline & $\begin{array}{l}\text { \# of stimulation } \\
\text { sessions }\end{array}$ & $\begin{array}{l}\text { \# of Ml } \\
\text { neurons }\end{array}$ & $\begin{array}{l}\text { \# of GPe } \\
\text { neurons }\end{array}$ & $\begin{array}{l}\text { \# of GPi } \\
\text { neurons }\end{array}$ \\
\hline $\begin{array}{c}\text { Monkey T } \\
\text { CTX stim. }\end{array}$ & $54 \mathrm{~N}, 55 \mathrm{P}$ & $146 \mathrm{~N}, 166 \mathrm{P}$ & $57 \mathrm{~N}, 143 \mathrm{P}$ & $9 \mathrm{~N}, 34 \mathrm{P}$ \\
GP stim. & $62 \mathrm{~N}, 66 \mathrm{P}$ & $238 \mathrm{~N}, 259 \mathrm{P}$ & $46 \mathrm{~N}, 104 \mathrm{P}$ & $3 \mathrm{~N}, 42 \mathrm{P}$ \\
Monkey W & & & & \\
CTX stim. & $79 \mathrm{~N}, 23 \mathrm{P}$ & $184 \mathrm{~N}, 88 \mathrm{P}$ & $99 \mathrm{~N}, 86 \mathrm{P}$ & $36 \mathrm{~N}, 10 \mathrm{P}$ \\
GP stim. & $48 \mathrm{~N}, 17 \mathrm{P}$ & $197 \mathrm{~N}, 107 \mathrm{P}$ & $47 \mathrm{~N}, 48 \mathrm{P}$ & $27 \mathrm{~N}, 3 \mathrm{P}$ \\
\hline
\end{tabular}

A summary of the number of stimulating sessions (left column) and the number of $\mathrm{Ml}, \mathrm{GPe}$, and GPi neurons that were recorded while stimulating in each of the areas. Neurons included here are those satisfying the inclusion criteria for analysis (see Materials and Methods). Number of stimulating sessions and recorded neurons is given for normal and MPTP states. N, Normal state; P, MPTP state.

Table 2. Summary of cortical stimulating sessions that produced movements

\begin{tabular}{lll}
\hline & $\begin{array}{l}\text { \# of sessions that produced movement/ } \\
\text { \# of all sessions (percentages) }\end{array}$ \\
\cline { 2 - 3 } & Normal state & MPTP state \\
\hline Monkey T & $34 / 54(63 \%)$ & $20 / 55(36 \%)$ \\
Monkey W & $49 / 79(62 \%)$ & $14 / 23(60 \%)$ \\
\hline
\end{tabular}

A summary of number (and percentage) of cortical sessions that produced an arm movement that was recorded by the accelerometer out of the total number of cortical stimulating sessions, in both normal and MPTP states. We defined a session with a clear and robust response to at least a single frequency test as a session that produced movement.

min of her death. Brains were removed and cryoprotected in increasing gradients of sucrose (10,20, and finally $30 \%)$. Adjacent serial sections of $50 \mu \mathrm{m}$, from both control animals and MPTP-treated animals, were processed for either a Nissl stain or immunocytochemistry for tyrosine hydroxylase (TH). Sections were incubated with antisera to TH (mouse anti-TH, 1:20,000; Eugene Tech, Allendale, NJ) in $0.1 \mathrm{M}$ phosphate buffer with $0.3 \%$ Triton X-100 and 10\% normal goat serum (Incstar, Stillwater, $\mathrm{MN}$ ) for 4 nights at $4^{\circ} \mathrm{C}$ and further processed using the avidin-biotin method (rabbit Elite Vectastain ABC kit; Vector Laboratories, Burlingame, CA).

\section{Results}

\section{Animals' clinical states}

We recorded from the arm area of the primary motor cortex and the globus pallidus of two vervet monkeys ( $\mathrm{T}$ and W). After 24 and 19 recording days in the normal state for monkeys $\mathrm{T}$ and $\mathrm{W}$, respectively, the monkeys were systemically treated with the neurotoxin MPTP.

The first signs of Parkinsonism appeared within $5 \mathrm{~d}$ from initiation of the MPTP treatment in monkey $\mathrm{T}$, and included muscle rigidity, akinesia and bradykinesia. Five days later, the monkey developed a low-frequency episodic tremor, mainly in the distal limb muscles. We started the recordings of monkey $\mathrm{T}$ in the MPTP state on the 11th day after the first MPTP injection and the monkey remained in a stable condition of severe Parkinsonism during all recording days with an average Parkinsonism score of 19.7/25 (Benazzouz et al., 1995). Dopamine replacement therapy started $21 \mathrm{~d}$ after the last MPTP injection and the first response to therapy was seen within $24 \mathrm{~h}$. The dopamine therapy effects included the resumption of the ability to self-feed, straightening of posture, and an increase in the amount and velocity of movement. The dense TH immunoreactivity throughout the striatum of the control monkey (Fig. 1D) was not observed in the striatum of Monkey $\mathrm{T}$, with the exception of the shell of the ventral striatum (Fig. $1 E$ ). Cell loss in the midbrain was almost complete in the ventral tier and the lateral portions of the substantia nigra pars compacta. As expected (Song and Haber, 2000), the cells in the ventral tegmental area of the midbrain dopamine system remained relatively spared. 
Table 3. Summary of average firing rates in the cortex and pallidum before and after MPTP treatment

\begin{tabular}{|c|c|c|c|c|c|c|}
\hline & \multicolumn{2}{|l|}{ CTX } & \multicolumn{2}{|l|}{ GPe } & \multicolumn{2}{|l|}{$\mathrm{GPi}$} \\
\hline & Normal & MPTP & Normal & MPTP & Normal & MPTP \\
\hline Monkey T & $\begin{array}{l}5.3 \pm 6.2 \\
(n=384)\end{array}$ & $\begin{array}{c}7.1 \pm 9.0 \\
(n=425)^{* *}\end{array}$ & $\begin{array}{c}64.5 \pm 30.9 \\
(n=103)\end{array}$ & $\begin{array}{l}49.7 \pm 28.9 \\
(n=247)^{* * *}\end{array}$ & $\begin{array}{c}69.1 \pm 27.4 \\
(n=12)\end{array}$ & $\begin{array}{c}66.8 \pm 28.5 \\
(n=76)\end{array}$ \\
\hline Monkey W & $\begin{array}{l}5.3 \pm 7.0 \\
(n=381)\end{array}$ & $\begin{array}{l}4.3 \pm 6.4 \\
(n=195)\end{array}$ & $\begin{array}{c}68.3 \pm 33.7 \\
(n=146)\end{array}$ & $\begin{array}{l}51.7 \pm 27.4 \\
(n=134)^{* * *}\end{array}$ & $\begin{array}{c}71.8 \pm 32.6 \\
(n=63)\end{array}$ & $\begin{array}{c}59.8 \pm 20.8 \\
(n=13)\end{array}$ \\
\hline
\end{tabular}

Discharge rates (spikes/s) are given as mean \pm SD for MI, GPe, and GPi neurons. The rates are calculated based on the IStiml periods between the different frequency tests. We used the $10 \mathrm{~s}$ epochs that start $5 \mathrm{~s}$ after the last burst of the stimulation. $n$ stands for the number of neurons on which the values are based. ${ }^{* *} p<0.01,{ }^{* * *} p<0.001$ significant differences of MPTP values versus corresponding normal values.

Monkey $\mathrm{W}$ developed akinesia and bradykinesia, as well as prolonged episodes of low-frequency tremor within $5 \mathrm{~d}$ from the first MPTP injection. On the eighth day, monkey W was moderately rigid. Recordings from monkey W in the MPTP state started on the seventh day after the first injection. The average Parkinsonism score of monkey W was 15.1/25 during the MPTP recording days. We lost monkey W unexpectedly $11 \mathrm{~d}$ after the start of the MPTP injections, after $5 \mathrm{~d}$ of recordings.

\section{Experimental protocols}

During the experiment, we stimulated either in MI or in the GP in each stimulation session, while recording the evoked neural activity in those areas as well as the contralateral arm movements. The stimulation pattern consisted of 35 ms bursts ( 8 pulses at 200 $\mathrm{Hz}$ ) given at different frequencies (for details, see Materials and Methods). Table 1 summarizes the number of stimulating sessions and the number of recorded neurons in each monkey, state and area. Table 2 summarizes the total number and the fraction of MI stimulation sessions that led to arm movement.

Table 3 summarizes the average firing rates of the neurons calculated at the periods between the stimulations (IStimI). In line with previous studies, after MPTP a significant (Student's $t$ test, $p<0.001$ ) decrease in the discharge frequency of GPe neurons, but not in GPi neurons, was observed (Bergman et al., 1994; Boraud et al., 1998; Raz et al., 2000; Heimer et al., 2002). We did not observe a significant difference in the discharge rate of monkey W's MI neurons [also consistent with previous studies (Doudet et al., 1990; Goldberg et al., 2002)], although the discharge rate of MI neurons of monkey $\mathrm{T}$ increased slightly after $\operatorname{MPTP}(p<0.01)$.

\section{Responses of single neurons in the frequency domain}

Many of the MI and GP neurons responded to MI and/or GP stimulation. Some examples of single neuron responses to the different frequency tests are depicted in Figures 2 and 3.

Figure $2 \mathrm{~A}$ shows the response of a single neuron in the arm related area of the primary motor cortex to stimulation in that area in the normal state. Examples of the analog traces (bandpass filtered) containing the responses to single bursts in the different frequency tests appear in the upper three rows. The raster plots and the PSTHs are shown in the lower two rows for each of the frequency tests. The neuron responded to each burst by a strong inhibition that lasted $>200 \mathrm{~ms}$. As a result, in the $5 \mathrm{~Hz}$ and in higher frequency tests the cell hardly produced any action potentials in the interburst periods. Many MI neurons responded to MI stimulation with decreases in their firing rates that often resulted in zero spikes for a certain period. Naturally, the duration of this silent period determined the stimulation frequency at which MI neurons stopped firing action potentials.

Another MI neuron that was recorded in the MPTP state (Fig. $2 B$ ) demonstrated a triphasic response to MI stimulation bursts: an initial short phase of elevation of the firing rate, followed by a $\sim 200$ ms of suppression of discharge rate, and a rebound excitation. The rebound excitation phase was attenuated in frequencies of $5 \mathrm{~Hz}$ and higher. Moreover, the first phase of excitation was also reduced as the frequency of stimulation increased, despite its short latency.

An example of the response of an MI neuron to GP stimulation in the MPTP state is illustrated in Figure 2D. The neuron responded by a sharp increase in firing rate that occurred $\sim 200$ $\mathrm{ms}$ after the end of the burst. As in the case shown in Figure $2 B$, the response was not seen in the $5 \mathrm{~Hz}$ and higher frequency tests.

Figure 3 depicts examples of GP responses to GP stimulation (Fig. $3 A, B$ ) and to MI stimulation (Fig. $3 C, D$ ). For example, the GPe neuron in Figure $3 A$ tended to increase its firing rate immediately after the GP microstimulation burst. In contrast with the long duration cortical responses, the response of this GPe neuron lasted $<50 \mathrm{~ms}$ after the burst, after which the neuron resumed its original firing rate. Furthermore, in contrast to the first excitation phase of the MI neuron (Fig. $2 B$ ) the increase in the firing rate of this GPe neuron did not change between the $1 \mathrm{~Hz}$ frequency test and the $10 \mathrm{~Hz}$ frequency test. The decrease seen in the PSTH of the $15 \mathrm{~Hz}$ frequency test is the result of the smoothing which was performed for purposes of illustration of the single cell PSTH (for this reason we did not use smoothing in the calculation of the population PSTH, see below). Indeed, the spike density as seen in the examples of the analog traces did not seem to decrease in the $15 \mathrm{~Hz}$ frequency test. This suggests that the responses of the GPe neuron in this example were not frequency dependent.

\section{MI population response to stimulation in the MI: normal and MPTP}

Figure $4 A$ shows the population response of neurons in the arm related area of the primary motor cortex to microstimulation in that area in the normal and MPTP states. The normalized population PSTH in both states shows that the response is attenuated as the frequency is increased (as in the single cell examples) (Fig. $2 A, B)$. The main difference between the normal and the MPTP states is the rebound excitation phase that occurs at the low frequencies ( 1 and $2 \mathrm{~Hz}$ stimulations) and is apparent only in the parkinsonian population.

The decrease-increase analysis revealed that different MI neurons did not tend to simultaneously increase or decrease their firing rate. Rather, the first bin in the decrease-increase plots reveals a small fraction of MI neurons with increases in firing rate, whereas decreases become dominant from the second bin on (Fig. 4A, bottom) (see Table 5). In the $1-2 \mathrm{~Hz}$ tests of the MPTP state, in which the rebound excitation is apparent $(\sim 250 \mathrm{~ms}$ after the burst), the fraction of decreases drops as the fraction of increases rises. The mean diversity index of the population (see Materials and Methods) was 0.15 and 0.35 in the normal and MPTP states, respectively (Table 4). These values indicate a relatively similar temporal profile of neuronal responses compared with the more divergent responses found in the GP neurons (see 


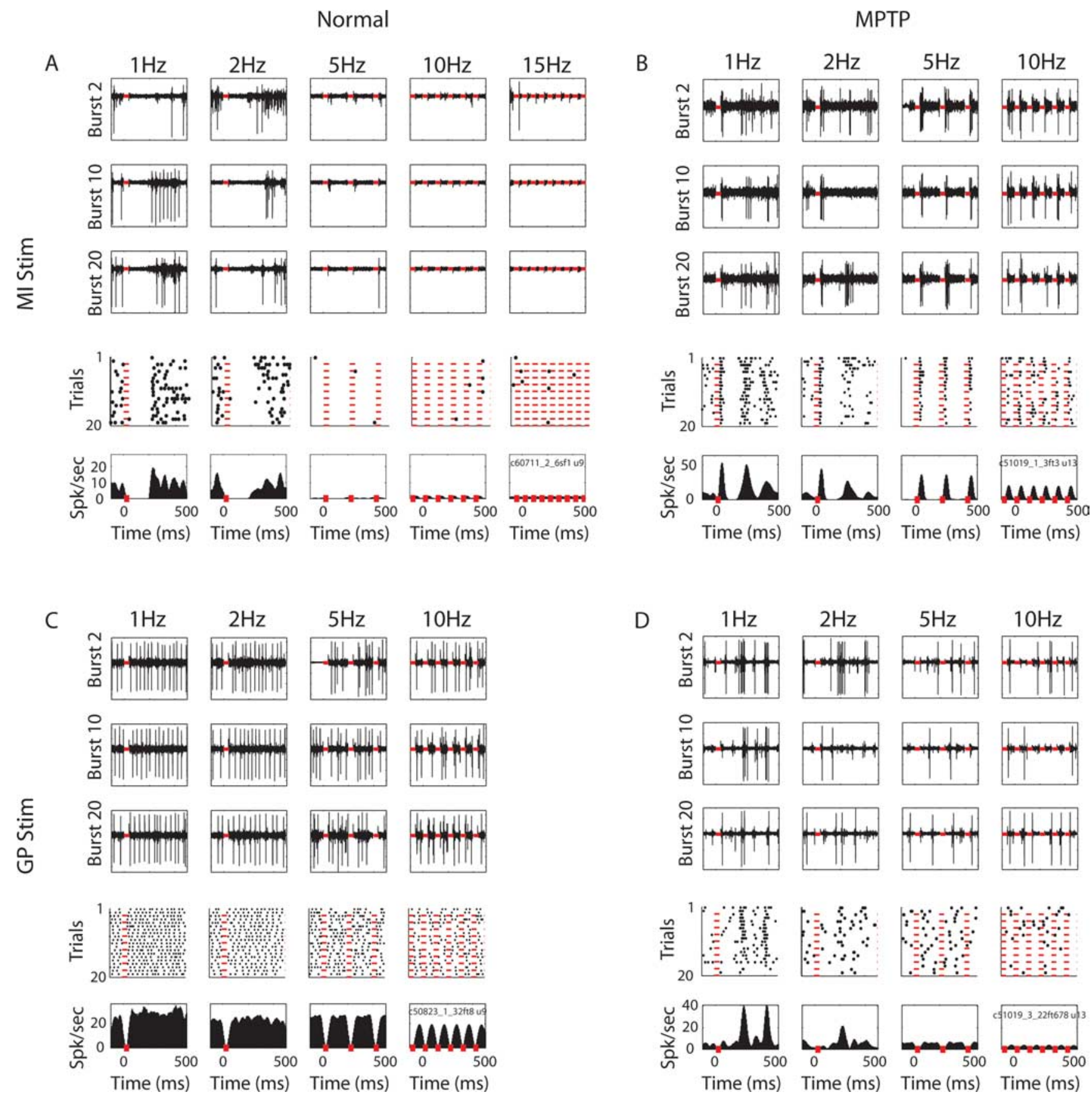

Figure 2. Responses of single primary motor cortex (MI) neurons to stimulation. $\boldsymbol{A}$, MI neuron response to Ml stimulation in the normal state. Three top rows, The bandpass filtered analog data recorded from an electrode located in MI while stimulating through another MI electrode in monkey W. First, second, and third rows show the response to the 2nd, 10th, and 20th bursts in each frequency test, respectively. All periods of stimulation bursts ( $40 \mathrm{~ms}$ per burst, to enable recovery from the last stimulus artifact) were off-line deleted and are marked by red lines. Fourth row, Raster displays of the neuron. Each dot in the raster represents an action potential of the neuron. For illustration purposes, in conditions with $>20$ trials (i.e., for $2 \mathrm{~Hz}$ and higher-frequency tests), only a subset of 20 equally distributed trials is shown in the raster plots. Bottom, The mean firing rates aligned on the stimulation bursts (PSTH). Bin size, 5 ms. PSTHs are smoothed with a Gaussian window, $\sigma=15 \mathrm{~ms}$. Note that the analog data recorded from the electrode reveal action potentials from more than a single neuron (see burst 2 of the $2 \mathrm{~Hz}$ frequency test), but here we focused on the cell with the highest signal-to-noise ratio. $\boldsymbol{B}$, MI neuron response to stimulation in Ml in the MPTP state of monkey T, same convention as $\boldsymbol{A}$. $\boldsymbol{C}, \boldsymbol{D}$, Responses of MI neurons to stimulation in GP in the normal and MPTP states, monkey T, same convention as $\boldsymbol{A}$.

below). Note that the fraction of increases in the early excitation phase is underestimated because of the $25 \mathrm{~ms}$ binning that blends the short period of increase in the firing rate (a few milliseconds) with the decrease that follows it. However, early excitation was found only in a small fraction of MI neurons and the majority of MI neurons in the normal state responded to MI stimulation with suppression of their discharge rate after the stimulation burst (Fig. 2A).

\section{MI population response to stimulation in the GP: normal} and MPTP

In the normal state, MI neurons were not affected by GP microstimulation (see example in Fig. $2 C$ ). This is confirmed by the population PSTH that does not change as a result of the stimulation bursts in the GP in the normal state (Fig. $4 B$, top, green line). The decrease-increase analysis indicates very small percentages of neurons that significantly modified their firing rates as a result 
Normal

MPTP
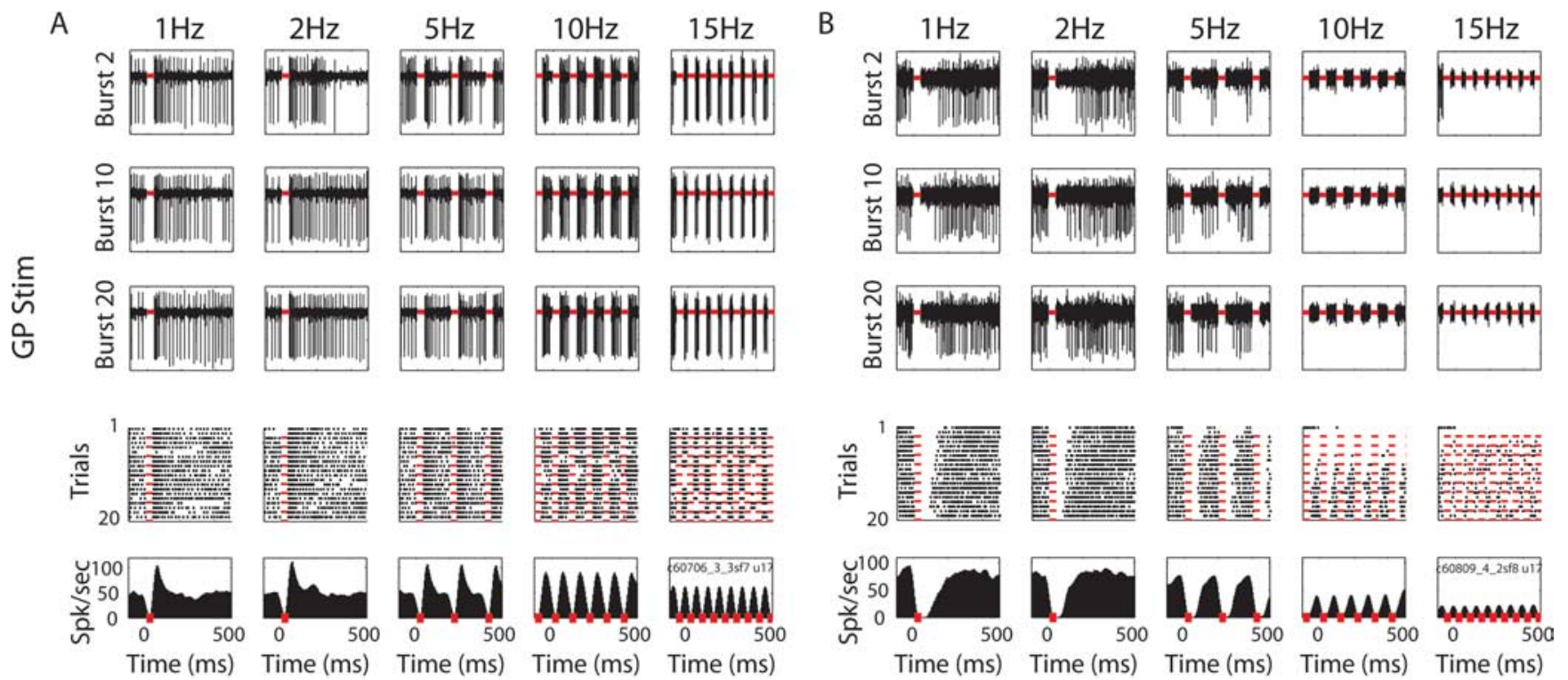

C
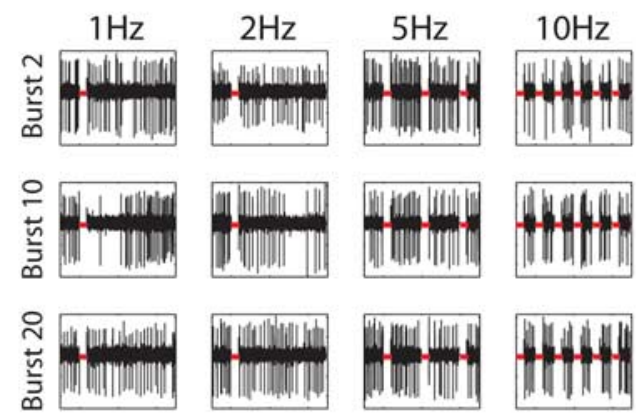

$\stackrel{\Xi}{\stackrel{E}{n}}$
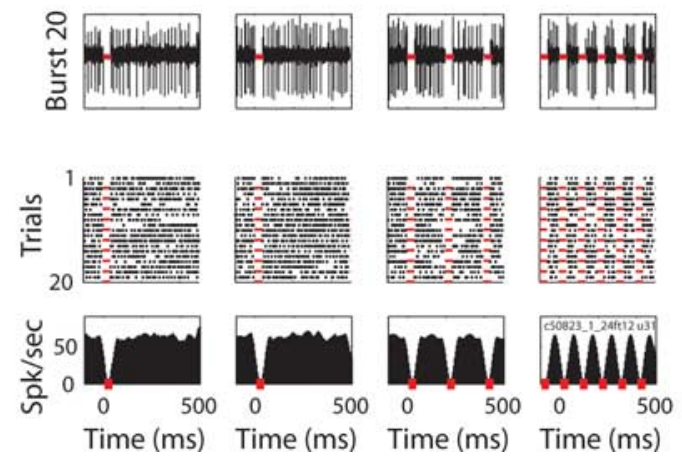

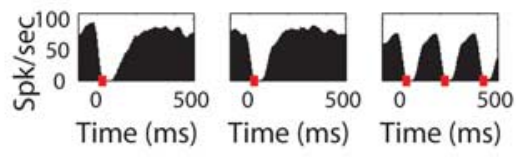

Time (ms)
D
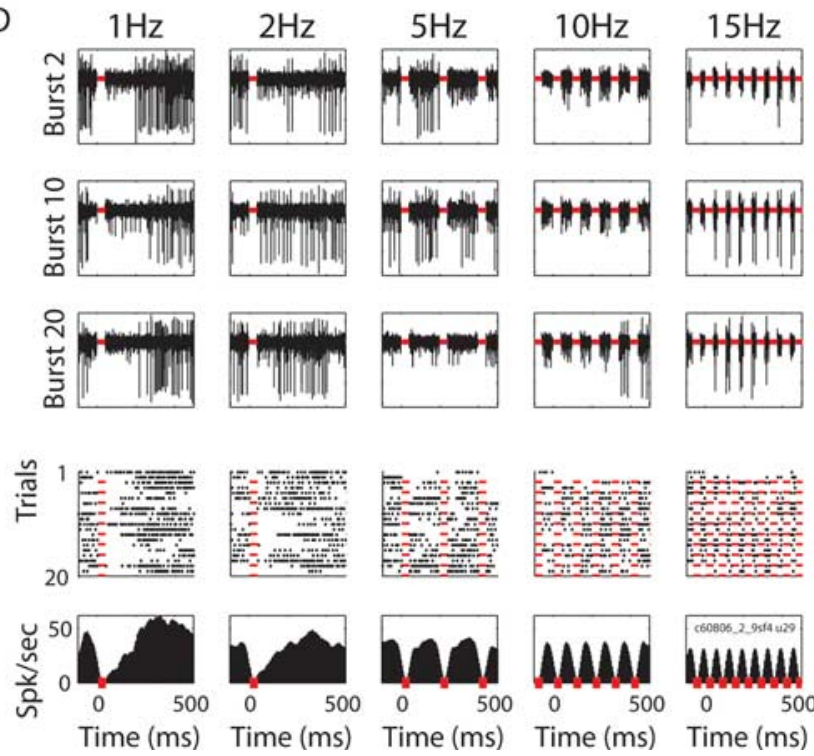

MUN

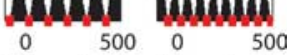

Figure 3. Responses of single pallidal neurons to stimulation. $A$, GPe neuron response to stimulation in GP in the normal state of monkey W. $B$, GPi neuron response to stimulation in GP in the MPTP state of monkey W. C, GPi neuron response to stimulation in Ml in the normal state of monkey T. D, GPe neuron response to stimulation in Ml in the MPTP state of monkey W. Conventions as in Figure $2 A$.

of the stimulation bursts (Fig. $4 B$, bottom, green line). Nonetheless, in the MPTP state, the efficacy of the functional connectivity between GP and MI appears to increase, and the MI neuron shown in the example (Fig. 2D), as well as the population as a whole (Fig. $4 B$, top, black line), do respond to the GP stimulation bursts. Although the MI neurons of both monkeys elevated their discharge rate after the GP stimulation, we noted a difference in the timing of these rate modulations (Table 5). The populations from the two monkeys in the MPTP state are therefore plotted separately. In both monkeys, this cortical response was attenuated from the $5 \mathrm{~Hz}$ frequency test and up. The decrease-increase analysis reveals that the MI population did not tend to opposing modulations in the firing rate in the MPTP state in response to the GP stimulation (Fig. $4 B$, bottom, black line). In accordance, the mean diversity in- dex demonstrates low values for both monkeys ( 0.26 and 0.31 for monkeys $\mathrm{T}$ and $\mathrm{W}$, respectively, vs 0.48 in the normal state) (Table 4). Despite the substantial cortical response observed in the population PSTHs of 1 and $2 \mathrm{~Hz}$ stimulations, the maximal fraction of neurons that responded with significant increases in the firing rate was $\sim 13 \%$. This may indicate that a small cortical area was activated by the microstimulation, and that despite the increase in the functional connectivity between the GP and the MI, a state of all-to-all connectivity was not attained.

GP population response to stimulation in the GP: normal and MPTP

The GP stimulation in both states produced various responses in the neurons of the GP. When examining the populations as a 

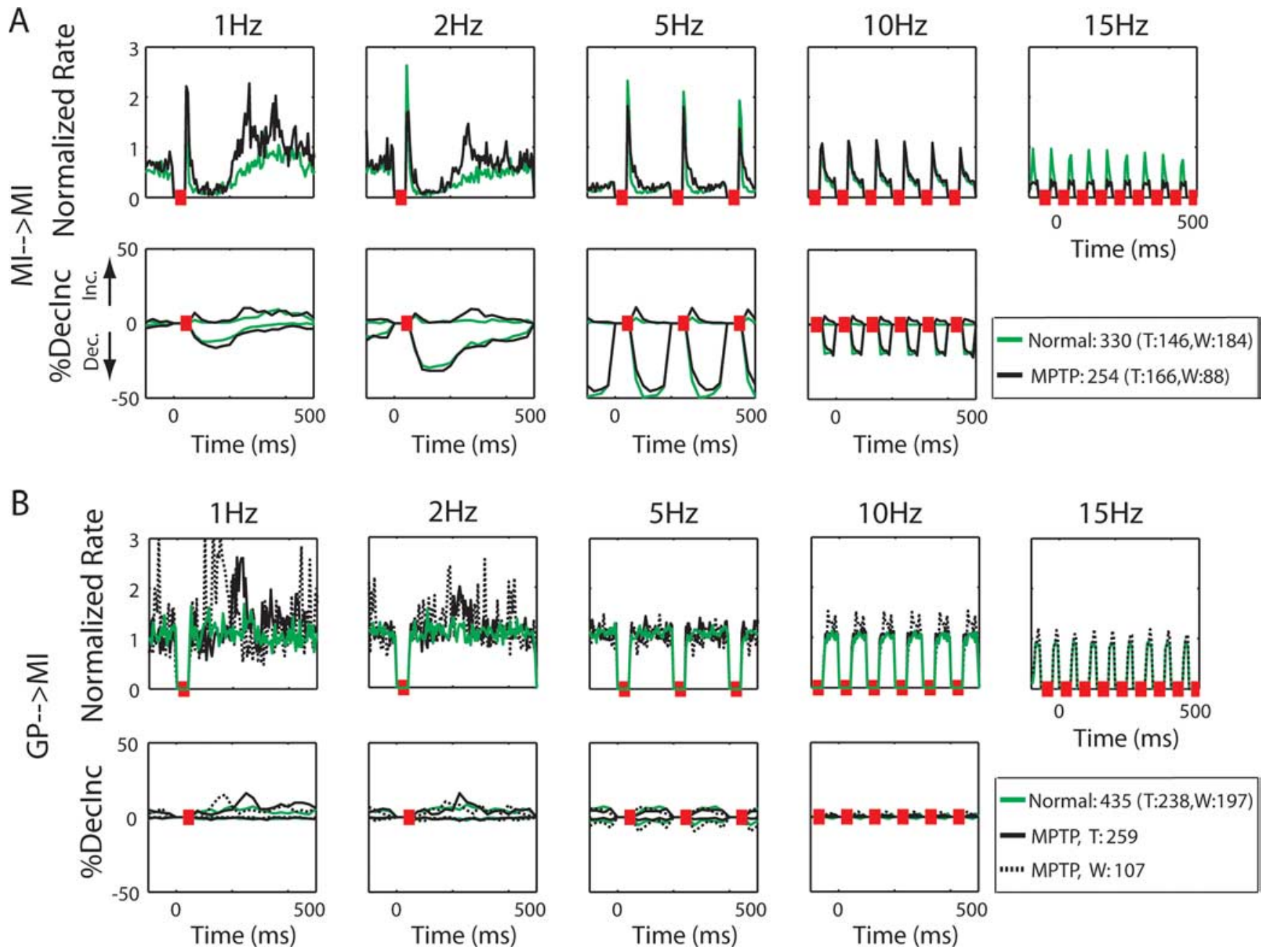

Figure 4. MI population response to stimulations. $\boldsymbol{A}$, MI population response to stimulation in MI in the normal and MPTP states. Top, The population PSTH of all recorded cortical neurons in response to cortical stimulation. PSTHs were normalized by the spontaneous firing rate of the cell (see Materials and Methods). Mean PSTH is shown as a green line for the normal state and as a black line for the MPTP state. Bottom, Fraction of cells with significant modulations in firing rate around the stimulation burst. The ordinate is the fraction of cells that had a significant response at each time bin (bin size was $25 \mathrm{~ms}$ in all frequencies except $10 \mathrm{~Hz}$ stimulation, in which bin size was $10 \mathrm{~ms}$ ). The values above 0 are the fraction of cells that increased their firing rate, and the values below 0 are the fraction of cells that decreased their firing rate. The total number of recorded neurons in each of the states is given in the legend, and the numbers in parentheses detail the number of neurons recorded from monkeys T and W separately. $\boldsymbol{B}$, MI population response to stimulation in GP in the normal and MPTP states. The green line represents the population of both monkeys in the normal state, black solid line represents the population of monkey T in the MPTP state, and dotted black line represents the population of monkey W in the MPTP state. All other conventions as in $\boldsymbol{A}$.

whole, GPe neurons responded with short increases in their firing rate (as in the example in Fig. $3 A$ ), but the decrease-increase analysis also reveals decreases of discharge rate (Fig. 5A; Table 5). The values of the diversity index were indeed higher than those in the cortex (mean values 0.79 and 0.77 for the normal and MPTP states, respectively) (Table 4). The different types of responses in the nonhomogenous GPe neural population counteract, and thus lead to the relatively small elevation seen in the averaged PSTH population. There was no noticeable change between the GPe response to GP stimulation in the normal and in the MPTP states. In both states the responses of the GPe neurons, increases as well as decreases, were independent of the frequency of the stimulation burst (see also the single GPe neuron example in Fig. 3A).

In contrast to the short and frequency-independent GPe responses, GPi neurons revealed a mixed frequency response in the normal state. The GPi neurons tended to have long decreases in their firing rates immediately after the stimulation burst, as well as delayed increases in the lower frequency tests (Fig. 5B, green lines; Table 5). As opposed to the GPe population, in which increases and decreases appeared simultaneously, the increases and decreases in the GPi were rarely coincident, as is reflected as well by the diversity index (mean value, 0.29 ) (Table 4 ). In the parkinsonian state, the responses of GPi neurons became more divergent, and early increases were observed together with decreases (Fig. $3 B$ ) in all frequency tests (Fig. $5 B$, black lines), and mean diversity index rose to 0.57 (Table 4). Nevertheless, GPi neurons showed prolonged responses to GP stimulation in the MPTP state, as in the normal state.

The divergent responses of the GP neurons relative to the MI neurons could be attributed to their high frequency tonic discharge rate, enabling them to respond either by an increase or a decrease of their discharge rate. Such response diversity is in line with intrinsic neuronal mechanisms directed at maximizing the information capacity of the pallidal network (Bar-Gad et al., 2003), whereas the cortical network is more redundant (Ben Shaul et al., 2003).

\section{GP population response to stimulation in the MI: normal} and MPTP

Figure 5, $C$ and $D$, illustrates the population response of GPe and GPi neurons to stimulation in the MI. Consistent with the 
absence of effect of GP stimulation on MI neurons in the normal state, the population PSTH confirms that in the normal state GP neurons in both segments did not change their discharge rate as a result of microstimulation in MI (see the single cell example in Fig. $3 C$; population analysis in Fig. 5C,D, green lines). The decrease-increase analysis confirms that the flat GP responses are not attributable to balanced positive and negative responses. However, minor changes that include both increases and decreases are seen mainly in the $5 \mathrm{~Hz}$ test, which might reflect some resonance properties of this pathway at this frequency. In the MPTP state, cortical microstimulation did affect the discharge of GP neurons. Although the GPe population PSTH did not significantly differ from the normal state (Fig. $5 C$, black lines), a small fraction of the recorded GPe cells did respond to the MI stimulation (Fig. 3D). Moreover, the GPi neuronal population exhibited enhanced functional connectivity with the MI as revealed by the increases in its mean firing rates as a result of MI microstimulation at 1 and $2 \mathrm{~Hz}$ (Fig. 5D, black lines; Table 5). The mean diversity indexes had similar values for both segments of the GP in each of the states (Table 4), and a reduction in the diversity of GP responses to MI stimulation was observed after the MPTP treatment. We did not observe phase-locking of the discharge of pallidal neurons as a result of the cortical stimulation. This contrasts with the long-lasting oscillatory GPi activity observed after striatal stimulation (albeit with a much higher current, $500 \mu \mathrm{A}$ ) in the MPTPtreated monkey (Tremblay et al., 1989).

\section{Muscle response to stimulation in MI: normal and MPTP}

Many of the cortical microstimulations produced forearm, wrist or finger movements (Table 2). Whether a movement occurred was a function of many factors, including the exact $\mathrm{X}-\mathrm{Y}$ coordinates of the stimulation (center of the arm related motor cortex or periphery), electrode depth, the current amplitude (ranged 40-60 $\mu$ A for each stimulating electrode), and the alertness of the animal [a stimulation that has been shown to elicit a response in an animal while alert may not elicit any response when the animal is drowsy (Alexander and DeLong, 1985)]. A few stimulations led to small thumb movements that were not recorded by the accelerometer which was located on the back of the wrist. These stimulations were categorized as no-movement stimulations.

Figure 6, $A$ and $B$, shows examples of the recorded acceleration of the hand in response to microstimulation in the MI in the normal and MPTP states. A reduction in the movement amplitude is seen in both examples in the frequency tests of $10 \mathrm{~Hz}$ and higher. The single burst examples reveal that although there was movement at the beginning of stimulation test (burst 2) in these frequencies, it rapidly decayed. The 2 and $5 \mathrm{~Hz}$ frequency tests, on the contrary, produced a movement that became stronger with time, as can be seen by comparing the single bursts with the normalized mean of all bursts (ACC PSTH). These examples are in line with the population average, which exhibited a substantial response for the $1 \mathrm{~Hz}$ stimulation, and greater response for the 2 and $5 \mathrm{~Hz}$ stimulations. A steep decay is seen in the $10 \mathrm{~Hz}$ frequency test, and there was no movement in response to the $15 \mathrm{~Hz}$ MI frequency test (Fig. 6C).

\section{Muscle response to stimulation in GP: normal and MPTP}

There was no muscle response to stimulation in the GP in the normal state (in agreement with Horak and Anderson, 1984). However, in the MPTP state, a small reaction was seen in the population average in frequency tests of 1 and $2 \mathrm{~Hz}$, for both monkeys (data not shown). This movement could have been the outcome of the stronger connectivity between GP and MI in the MPTP state, as derived from the cortical neural response to GP stimulation (Figs. 2D, 4B).

\section{The temporal profile of the neural and muscle responses to stimulation bursts}

The duration of each frequency test was $20 \mathrm{~s}$, resulting in a different number of bursts for each of the frequency tests. Our findings indicate low-pass filter properties in the cortex and GPi, in which the responses decay with the increasing frequency stimulation. However, this decrease may be the outcome of the growing number of stimulations rather than the stimulation frequency. For this reason, we averaged the responses of the populations to each burst separately in each of the frequency tests, and looked at the mean normalized firing rate in the $25 \mathrm{~ms}$ epoch that followed the burst as a function of the number of bursts. We chose a period of $25 \mathrm{~ms}$ because this is the minimal common period between bursts (determined by the $15 \mathrm{~Hz}$ frequency test, after removing the stimulation period) for all frequency tests. The results of this analysis are shown in Figure 7. The neuronal responses to the different frequency tests of almost all pathways studied were independent of the number of bursts. An exception is the MI population whose responses to the first 20 stimulations in the MI differed for the low- $(1,2 \mathrm{~Hz})$ and high- $(5,10$, and $15 \mathrm{~Hz})$ frequency tests. The latter show a considerable elevation of their initial responses that decayed immediately (within the first 20-30 bursts), as opposed to the stable response in the low frequencies. A similar analysis of the arm acceleration revealed that the muscle responses to the high-frequency tests but not to the lowfrequency tests also decayed with the number of the bursts (Fig. $6 A, B$, responses to bursts 10 and 20 vs response to burst 2 in the 10 and $15 \mathrm{~Hz}$ frequency tests). However, the slope of the arm acceleration curve was more moderate than the slope of the MI population, especially in the $5 \mathrm{~Hz}$ stimulation. To summarize, MI 
Table 5. Main effects of microstimulation

\begin{tabular}{|c|c|c|c|c|c|c|c|c|}
\hline & \multicolumn{2}{|l|}{$1 \mathrm{~Hz}$} & \multicolumn{2}{|l|}{$2 \mathrm{~Hz}$} & \multicolumn{2}{|l|}{$5 \mathrm{~Hz}$} & \multicolumn{2}{|l|}{$10 \mathrm{~Hz}$} \\
\hline & Dec & Inc & Dec & Inc & Dec & Inc & Dec & Inc \\
\hline \multicolumn{9}{|l|}{$\mathrm{CTX} \rightarrow \mathrm{CTX}$} \\
\hline \multicolumn{9}{|l|}{ Normal } \\
\hline \%Respond & 12.5 & 2.3 & 29.9 & 5.3 & 49.6 & 3.4 & 19.7 & 0.4 \\
\hline Latency (ms) & 125 & 75 & 125 & 75 & 100 & 75 & 60 & 60 \\
\hline Duration (ms) & 200 & 50 & 225 & 50 & $>200$ & 50 & $>100$ & 30 \\
\hline \multicolumn{9}{|l|}{ MPTP } \\
\hline \%Respond & 16.6 & $6.9 / 10.1^{a}$ & 31.8 & $6.5 / 9.7^{a}$ & 45.6 & 10.6 & 20.2 & 5.5 \\
\hline Latency (ms) & 150 & $75 / 250^{a}$ & 125 & $75 / 275^{a}$ & 125 & 75 & 90 & 60 \\
\hline Duration (ms) & 200 & $50 / 275^{a}$ & 250 & $50 / 100^{a}$ & $>200$ & 50 & $>100$ & 30 \\
\hline \multicolumn{9}{|l|}{$\mathrm{GP} \rightarrow \mathrm{CTX}$} \\
\hline \multicolumn{9}{|l|}{ Normal } \\
\hline \multicolumn{9}{|l|}{ MPTP } \\
\hline \%Respond & & $16.1 / 15.3^{b}$ & & $16.1 / 10.6^{b}$ & & & & \\
\hline Latency (ms) & & $250 / 175^{b}$ & & $225 / 175^{b}$ & & & & \\
\hline Duration (ms) & & $100 / 75^{b}$ & & $100 / 100^{b}$ & & & & \\
\hline \multicolumn{9}{|l|}{$\mathrm{GP} \rightarrow \mathrm{GPe}$} \\
\hline \multicolumn{9}{|l|}{ Normal } \\
\hline \%Respond & 19.4 & 32.3 & 26.9 & 31.2 & 33.3 & 33.3 & 24.7 & 29.0 \\
\hline Latency (ms) & 60 & 60 & 60 & 60 & 60 & 60 & 60 & 70 \\
\hline Duration (ms) & 110 & 90 & 70 & 120 & 10 & 60 & $>100$ & $>100$ \\
\hline \multicolumn{9}{|l|}{ MPTP } \\
\hline \%Respond & 24.3 & 22.4 & 38.1 & 25.0 & 44.7 & 27.0 & 38.9 & 27.0 \\
\hline Latency (ms) & 60 & 90 & 60 & 80 & 60 & 90 & 60 & 60 \\
\hline Duration (ms) & 40 & 140 & 90 & 140 & 90 & 110 & $>100$ & $>100$ \\
\hline \multicolumn{9}{|l|}{$\mathrm{GP} \rightarrow \mathrm{GPi}$} \\
\hline \multicolumn{9}{|l|}{ Normal } \\
\hline \%Respond & 60 & 13.3 & 66.7 & 16.7 & 76.7 & 26.7 & 73.3 & 3.3 \\
\hline Latency (ms) & 70 & 250 & 60 & 230 & 60 & 190 & 60 & $>100$ \\
\hline Duration (ms) & 70 & 30 & 80 & 140 & 60 & $>200$ & $>100$ & $>100$ \\
\hline \multicolumn{9}{|l|}{ MPTP } \\
\hline \%Respond & 64.4 & 26.7 & 66.7 & 31.1 & 57.8 & 24.4 & 40 & 22.2 \\
\hline Latency (ms) & 60 & 150 & 60 & 120 & 60 & 180 & 70 & 60 \\
\hline Duration (ms) & 50 & 360 & 50 & 230 & 50 & $>200$ & $>100$ & $>100$ \\
\hline \multicolumn{9}{|l|}{$\mathrm{CTX} \rightarrow \mathrm{GPe}$} \\
\hline \multicolumn{9}{|l|}{$\mathrm{CTX} \rightarrow \mathrm{GPi}$} \\
\hline \multicolumn{9}{|l|}{ Normal } \\
\hline \multicolumn{9}{|l|}{ MPTP } \\
\hline \%Respond & & 15.9 & & 13.6 & & & & \\
\hline Latency (ms) & & 120 & & 120 & & & & \\
\hline Duration (ms) & & 90 & & 140 & & & & \\
\hline
\end{tabular}

A summary of the main effects of microstimulation, including percentages of neurons responding by decrease (Dec) and by increase (Inc) in the firing rate, latencies (from beginning of the stimulation burst until the peak response), and durations of the responses. The values are given for each neural population and frequency stimulation, in each of the states (normal and MPTP). Details are given only for structures and stimulation frequencies with a significant number of responses, and populations that had no significant response in any of the frequencies are in bold.

${ }^{a}$ First and second values (separated by /) refer to the first excitation phase and to the rebound excitation phase, respectively.

${ }^{b}$ First and second values refer to monkeys $\mathrm{T}$ and W, respectively. In all other cases, no significant differences were found between monkeys $\mathrm{T}$ and $\mathrm{W}$, and their results are grouped.

neurons, as well as muscles, present a response that depends on the number of bursts when stimulating the MI with high-frequency bursts. This attenuation is in line with the low-pass properties of these pathways (see below). The responses of all other recorded populations were independent of the number of bursts and therefore are only dependent on the frequency of stimulation.

\section{Frequency-domain analysis of the basal} ganglia-cortex-muscle pathways

Figure 8 summarizes the frequency dependency of the responses of MI, GPe and GPi neurons to stimulation in each of the structures (MI and GP) in the frequency range tested in this study $(1-15 \mathrm{~Hz})$. The figure depicts the maximum value of the normalized population PSTH (Figs. 4,5 ) as a function of the stimulating frequency. The maximum absolute value of the normalized arm acceleration to MI stimulation (Fig. 6) is also depicted.

The responses of the GPe population to microstimulations in both MI and GP are independent of the frequency of the stimulation bursts (Figs. 5A, $C, 8 A$ ). In contrast, GPi neurons changed their responses as a function of the frequency. In particular, in the parkinsonian state, their firing rate was elevated in response to 1 and $2 \mathrm{~Hz}$ frequency tests, but not in the higher frequency tests (Figs. $5 B, D$, $8 A)$. MI neurons revealed more robust low-pass filter responses to their inputs. The response of the cortical neurons to MI stimulations decayed with frequency, such that hardly any activation was seen with burst stimulations of 10 and $15 \mathrm{~Hz}$ (Fig. 4A, $8 A$ ). The responses of the cortical neurons to stimulations in the GP had a lower cutoff frequency and responses to stimulations were no longer observed as of the $5 \mathrm{~Hz}$ frequency test (Figs. $4 \mathrm{~B}, 8 \mathrm{~A}$ ). Furthermore, MI stimulations of $10 \mathrm{~Hz}$ and higher frequencies were not transferred to the periphery, at least not at the level of movements recorded by the wrist accelerometer (Figs. 6, 8B).

\section{Discussion}

In this study, we explored the functional microconnectivity within the cortex-BG and cortex-periphery loops throughout the normal state and in MPTP-induced Parkinsonism. We recorded simultaneously the acceleration of the arm and the spik- 

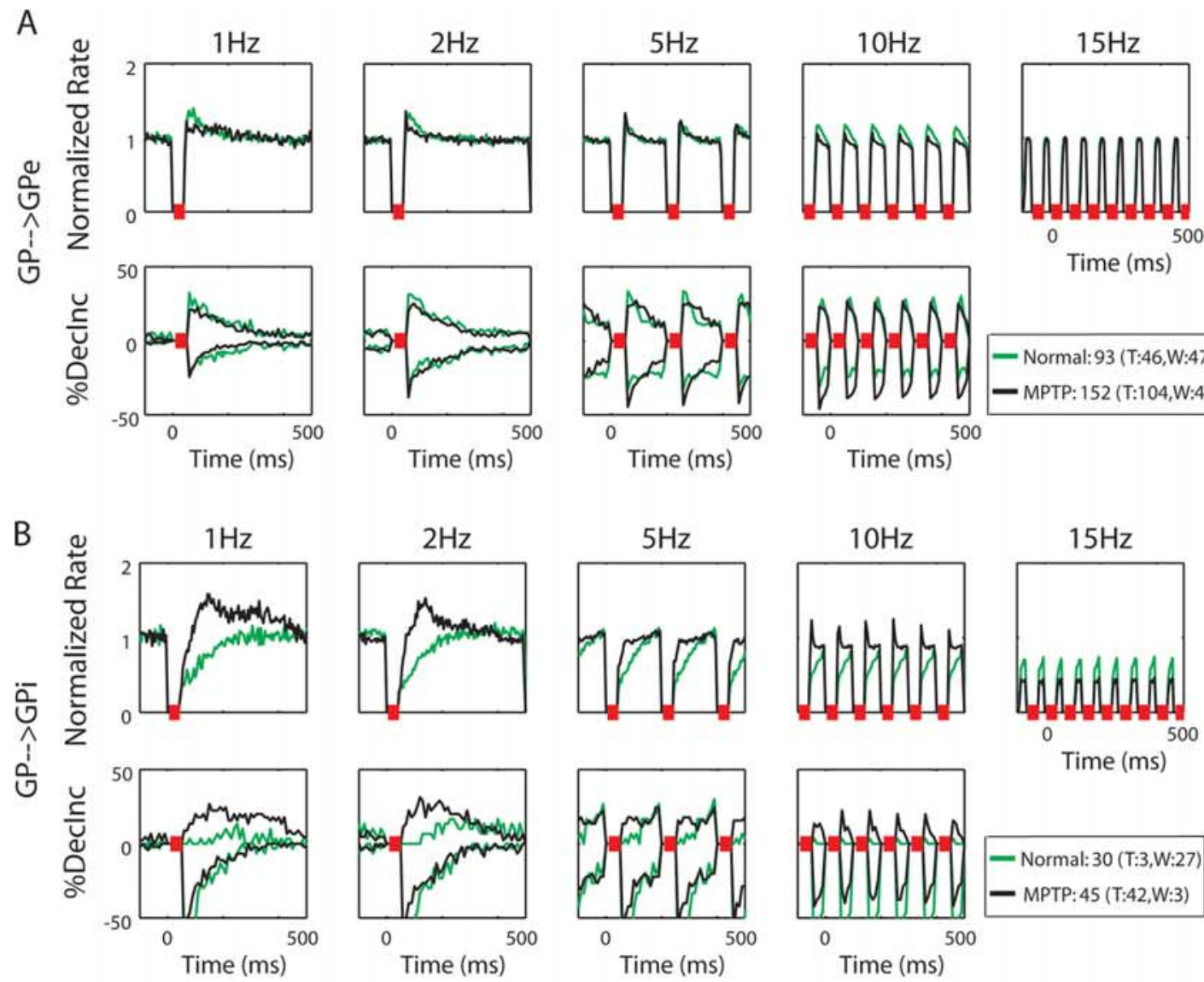

- Normal: 93 (T:46,W:47)
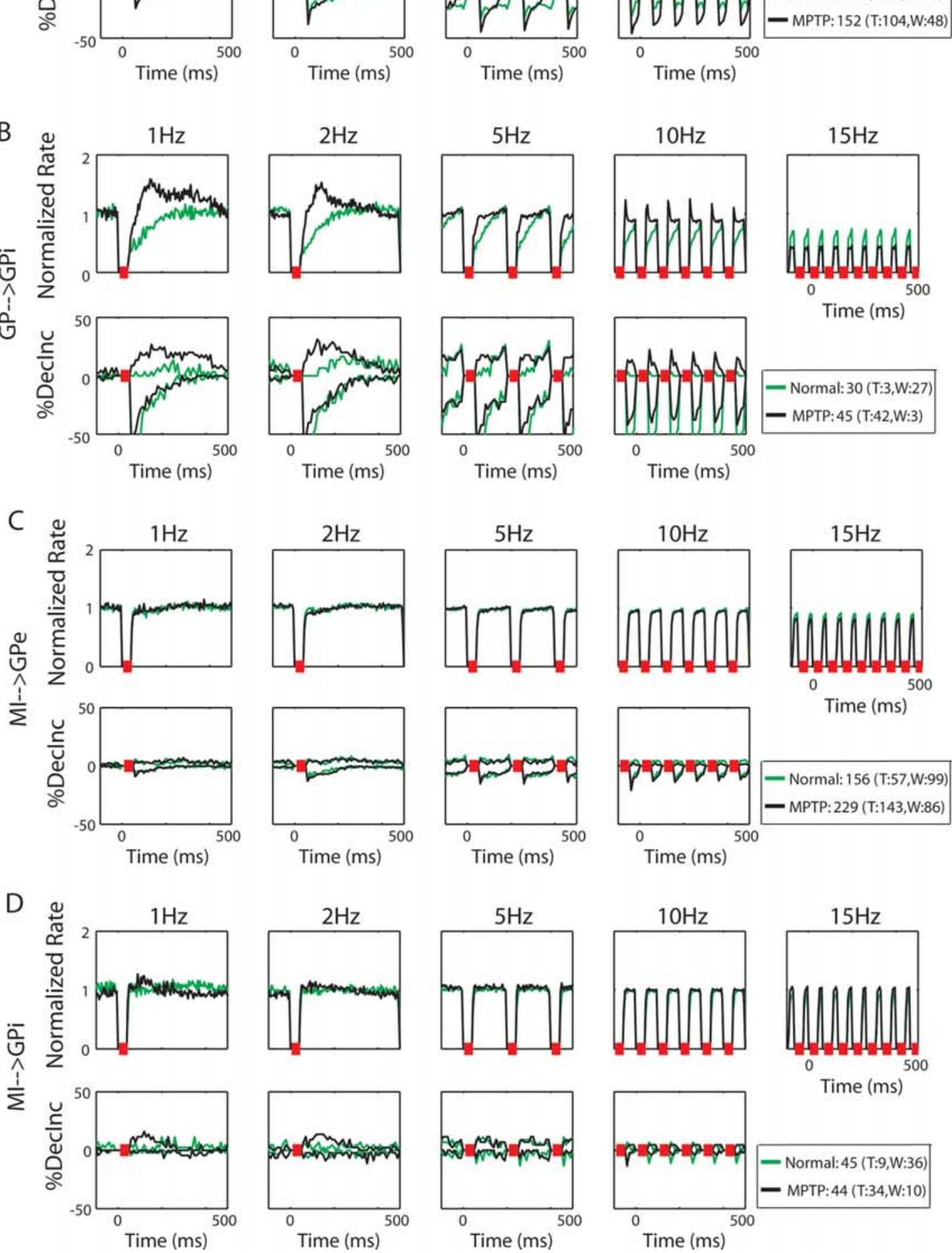

Figure 5. GP population response to stimulations. $A$, GPe population response to stimulation in GP in the normal (green) and MPTP (black) states. B, GPi population response to stimulation in GP in the normal and MPTP states. C, GPe population response to stimulation in MI in the normal and MPTP states. D, GPi population response to stimulation in MI in the normal and MPTP states. Conventions as in Figure $4 A$, except bin size, which was $10 \mathrm{~ms}$ for all frequencies. 
Normal

A
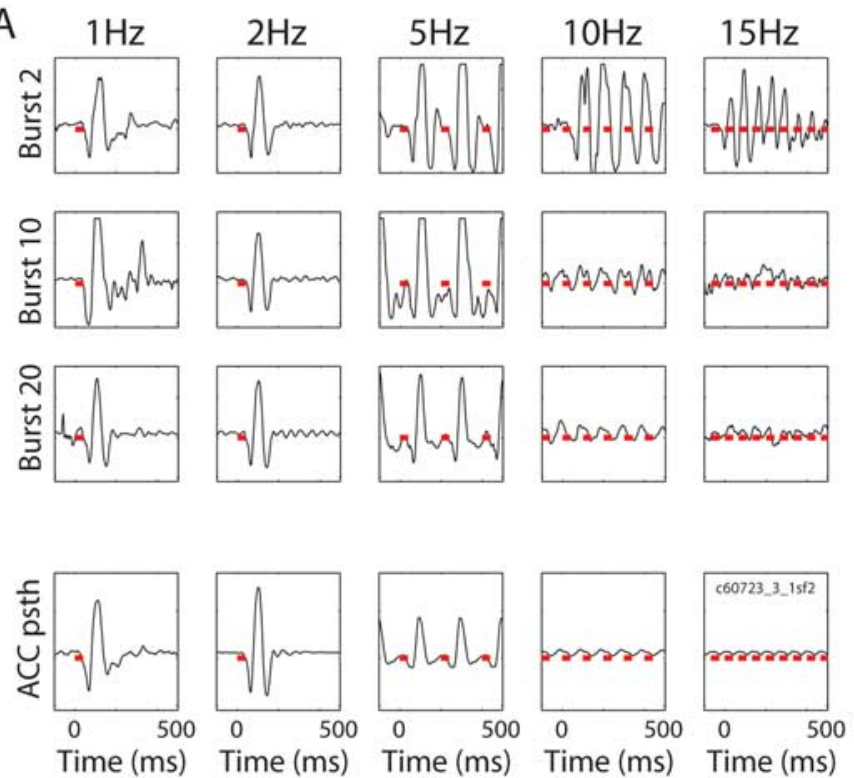

MPTP

B
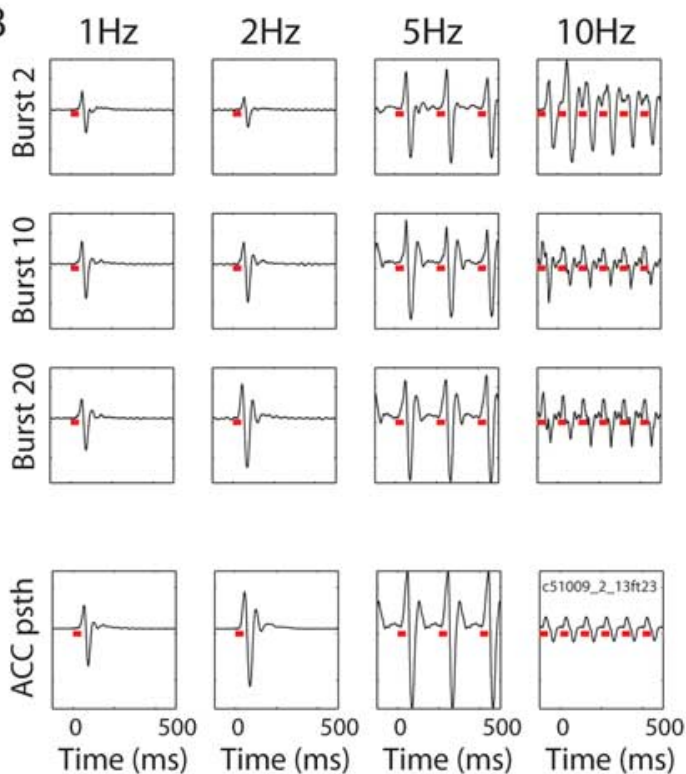
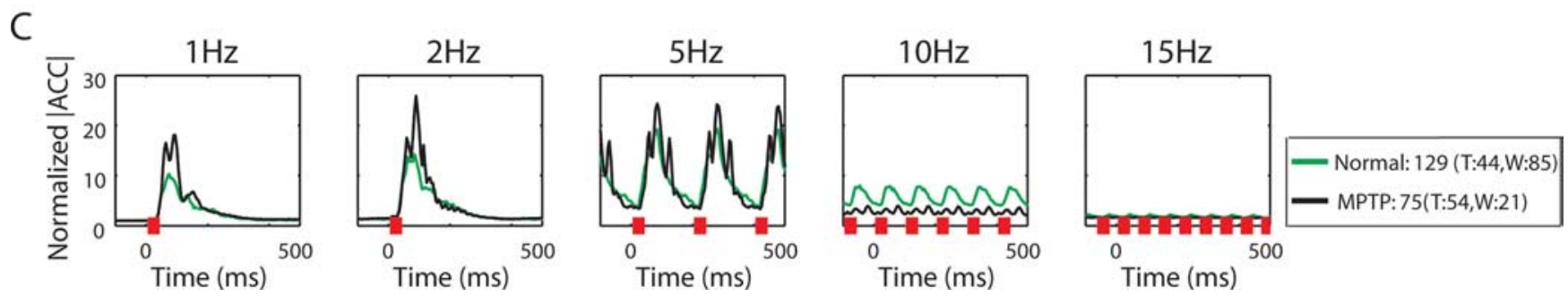

Figure 6. Arm acceleration as a result of MI microstimulation. $\boldsymbol{A}$, An example of the acceleration recorded from an accelerometer located on the back of the wrist while stimulating through an electrode in the contralateral motor cortex of monkey W in the normal state. First, second, and third lines show the responses to the 2nd, 10th, and 20th bursts in each frequency, respectively. All periods of stimulation bursts ( $40 \mathrm{~ms}$ per burst) are marked by red lines. Bottom, Mean acceleration values of the session aligned on the stimulation bursts. Ordinate units are arbitrary (A/D values). $\boldsymbol{B}$, An example of arm acceleration recorded during MI stimulations in monkey T in the MPTP state. Conventions as in $\boldsymbol{A}$. $\boldsymbol{C}$, The normalized population PSTH of the absolute acceleration values in response to MI stimulation in the normal and MPTP states. Only sessions in which MI stimulation evoked movements are included. The average of the normalized responses is shown in green for the normal state and in black for the MPTP state. Other conventions as in Figures 4 and 5.

ing activity from the contralateral MI arm related area and the GP of two vervet monkeys while microstimulating in one of the structures. To understand the role of oscillatory bursts encountered in the parkinsonian brain (Heimer et al., 2006; Weinberger et al., 2006) and the relationship between the GP, MI, and the periphery in the frequency domain, we mimicked the parkinsonian oscillatory activity using a stimulation pattern that contained $35 \mathrm{~ms}$ bursts delivered at different $(1-15 \mathrm{~Hz})$ frequencies. Because cortical neurons demonstrate bursting activity during normal movements (Georgopoulos et al., 1986; Evarts, 1966; Goldberg et al., 2002), the stimulation pattern also enabled us to characterize the transfer function between the MI and muscles. We report two main findings: first, the functional connectivity between the MI and the GP are greatly enhanced in the MPTP state. Second, in both the normal and the MPTP states, the BGMI-muscle circuit demonstrates low-pass filter properties.

\section{Functional connectivity between MI and GP}

The functional microconnectivity between MI and both segments of the GP is weak in the normal state. A comparison of our findings and previous reports of extensive GP activation by macrostimulation of MI (Nambu et al., 2000) suggests that in the normal monkey the MI to GP functional connectivity is either very specific or that converging inputs from many MI areas are needed to activate GP neurons. Had we focused on the hand related area of the GP, it is plausible that the results would have demonstrated stronger connections between the structures. Nevertheless, the MI-GP reciprocal connections are strengthened in the MPTP state. These findings are not attributable to increased excitability of the parkinsonian MI, because cortical discharge rate and the fraction of stimulating sessions that produced movement did not increase after MPTP treatment. Rather, this enhancement in connectivity may have been caused by the reduction in the specificity of pallidal neurons (Filion et al., 1988), as well as by the increased synchronization of BG neurons in the dopamine depleted state (Bar-Gad et al., 2003; Strafella et al., 2005; Heimer et al., 2006), leading to widespread activation of the local effects of microstimulation.

\section{Low-pass filter properties of the}

BG-cortical-muscle network

The cortex-BG-periphery loops demonstrate low-pass filter properties to the microstimulation pattern. The finding that MI does not follow high-frequency stimulations given in the GP is not surprising because of the multisynaptic pathway between the structures, especially because it contains inhibitory components 

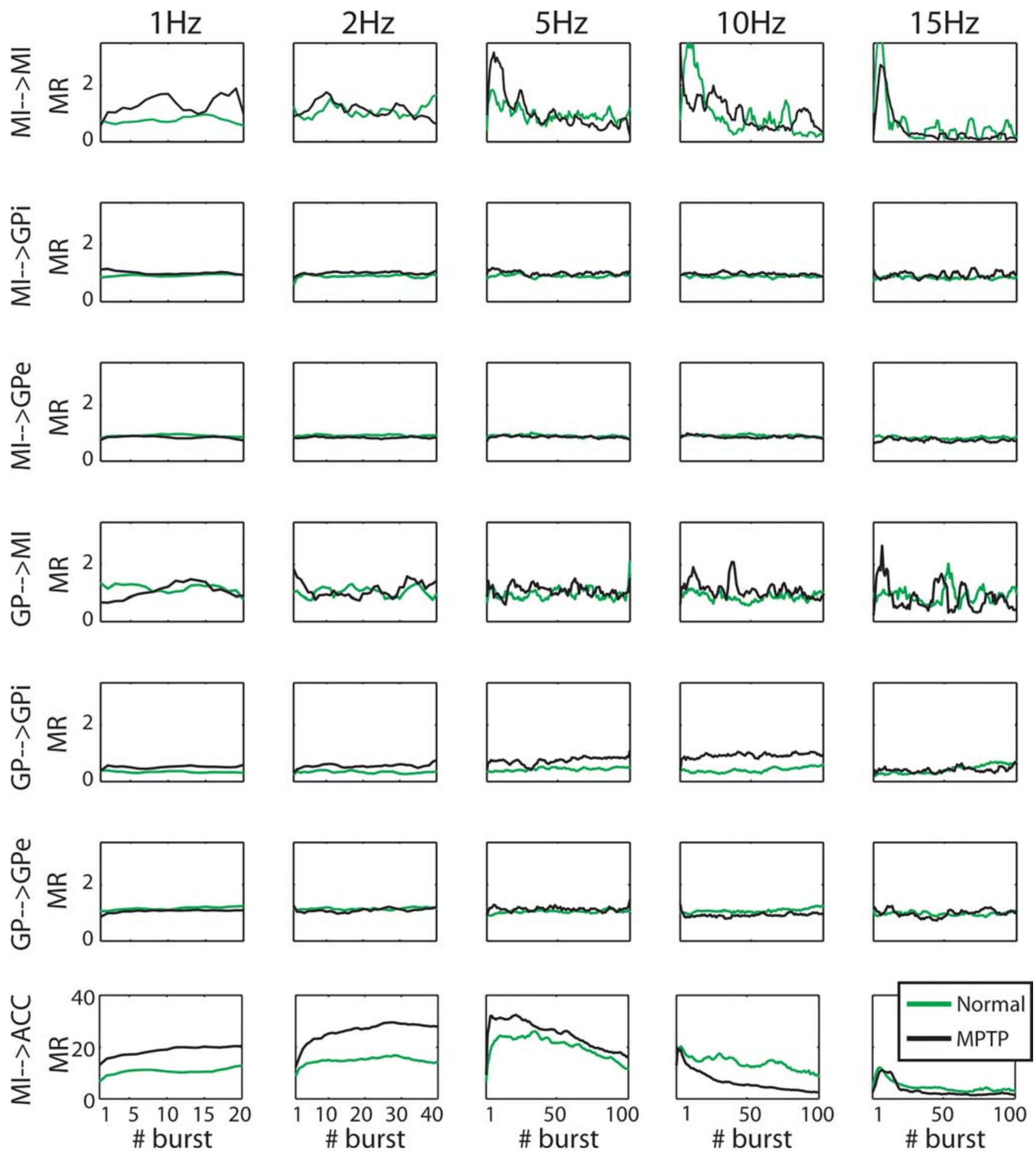

Figure 7. The neuronal response of most BG - cortex pathways to the different frequency tests are not affected by the number of bursts in the test. The mean normalized firing rate [mean response (MR)] in the $25 \mathrm{~ms}$ that follow each burst as a function of the sequential number of burst. For illustration purposes, only up to the first 100 bursts are shown. The last row illustrates the average of the maximum amplitude in the normalized absolute arm acceleration as a function of the number of burst. All curves were smoothed using a moving average of five bursts. Green and black lines represent normal and MPTP states, respectively.

(e.g., GPi to thalamus, and thalamus to inhibitory interneurons in the cortex). It has been suggested that BG $\beta$ - and $\gamma$-band oscillatory activity may arise in cortex (Hammond et al., 2007). The results of this study do not negate this possibility, because we did not find evidence for a prominent low-pass filter between MI and the two pallidal segments. Similarly, we do not expect the STN to demonstrate any substantial filtration properties.
The exact cutoff frequency of the different neuronal populations studied here is not known because we did not try all possible frequencies. Moreover, the cutoff frequency may change with the stimulation burst parameters [e.g., burst duration and frequency and number of the pulses/burst (McIntyre and Grill, 2002)]. Recent studies indicate a relationship between $\beta$-band oscillations and akinesia (Chen et al., 2007). Because the GP oscillatory bursts 
A
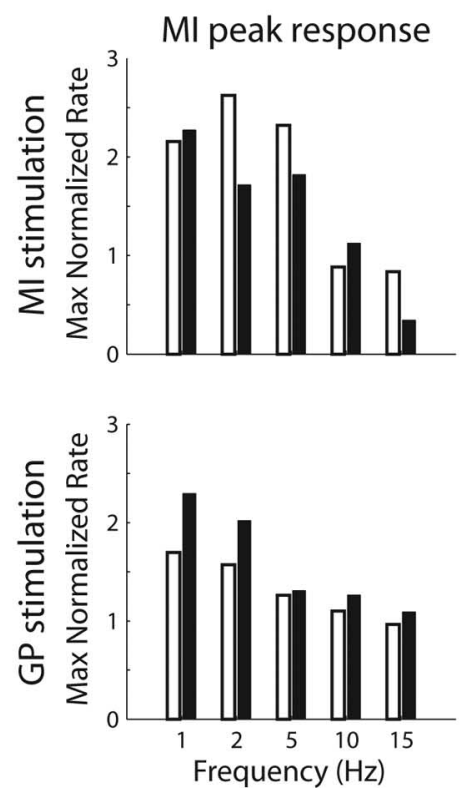

GPe peak response
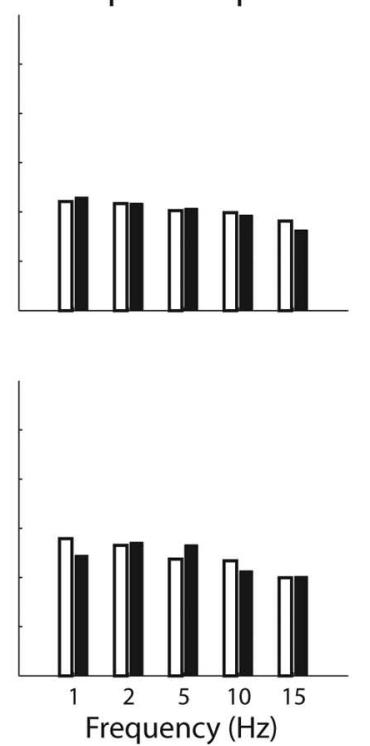

B
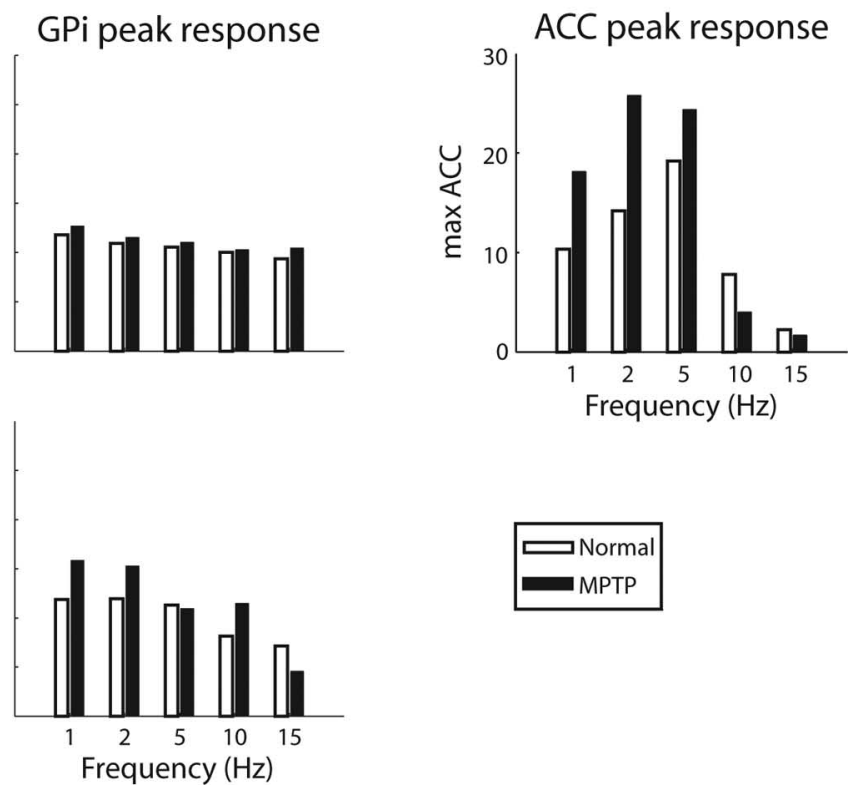

Figure 8. Frequency domain analysis of the BG- cortex-muscle network. $\boldsymbol{A}$, The maximum value of the normalized population PSTH of MI, GPi, and GPe neurons as a function of the stimulation frequency given in each of the structures (MI and GP). $B$, The maximum normalized absolute amplitude of the arm acceleration (ACC) as a function of the stimulation frequency in MI. A value of 1 stands for the mean ACC value in the "no movement" periods (when no stimulation occurred). Green and black bars correspond to the normal and MPTP states, respectively.

are non-stereotypical, their different characteristics may determine their filtration properties as well as their role in the generation of PD symptoms.

Low-pass filtering was also found between MI and the periphery level, in which MI stimulations of $10 \mathrm{~Hz}$ and higher did not evoke movement. Because we only recorded arm movements, we cannot rule out the possibility that this filtering is performed in muscle electrical-to-mechanical transfer. Indeed, many studies have reported significant coherence between MI and muscle EMG activity in the $\beta$ and even higher frequency ranges (Grosse et al., 2002). However, this cortex-EMG synchronization has mainly been detected during static motor tasks, and is reduced or abolished during movement (Baker et al., 1997; Kilner et al., 1999; Salenius and Hari, 2003). The low-pass filter properties of the entire network are in line with natural frequencies of movement $(\sim 2 \mathrm{~Hz})$ (Freund and Hefter, 1993), low-pass filter properties of striato-pallidal pathway (Rav-Acha et al., 2005) and nerve to muscle transmission (Baratta et al., 1998).

\section{DBS mechanisms in view of the low-pass filter properties of the network}

The low-pass properties of the GP-MI-periphery axis shed new light on the ongoing controversy regarding the mechanisms of high frequency $(\sim 130 \mathrm{~Hz})$ DBS in the treatment of advanced PD. There is considerable debate as to whether DBS mimics lesion and inactivates its targets (Wu et al., 2001; Filali et al., 2004; Maltete et al., 2007), or whether it drives them continuously at short latencies and higher frequencies (Hashimoto et al., 2003; Garcia et al., 2005). Because the bursts used in this study contained pulses given at $200 \mathrm{~Hz}$, we can view the 10 and $15 \mathrm{~Hz}$ frequency tests as a fragmented DBS stimulation pattern, enabling us to examine the effect of DBS without the distortion of stimulation artifact. Our results suggest that the continuous DBS mechanism would similarly be filtered in the cortex or in previous levels. Although filtered, DBS indirectly alleviates the parkin- sonian symptoms, probably by stopping (jamming) the abnormal oscillations of the BG (Benabid, 2003).

\section{Frequency dependency of tremor evoked by} cortical stimulation

A transcranial magnetic stimulation (TMS) study in which trains of repetitive stimulations at $10-30 \mathrm{~Hz}$ were delivered to the MI of normal subjects revealed that the subjects developed an oscillatory movement (tremor) (Topka et al., 1999). Interestingly, the tremor frequency $(4-7 \mathrm{~Hz})$ was independent of the TMS frequency. In addition, an earlier study showed that electrical stimulation of MI during neurosurgery at a frequency of $60 \mathrm{~Hz}$ evoked a $5 \mathrm{~Hz}$ tremor, whereas $1-7.8 \mathrm{~Hz}$ macrostimulation of the cortex resulted in movements of the same frequency as the stimulation (Alberts, 1972). Thus, movement evoked by $<10 \mathrm{~Hz}$ stimulation may directly originate in the MI, whereas the tremulous movement that appears after higher frequencies of magnetic or electricalstimulation of the cortex may be attributable to the abnormal synchronous activity of the cortex. We assume that this abnormal synchronization indirectly causes tremulous movements that are the result of diminished cortical control, and probably reflect resonance properties of brainstem-spinal and muscle networks.

\section{The relationship between PD tremor and BG oscillations}

The low-pass filter properties of the GP-MI-muscle networks described above have implications for our understanding of the pathophysiology of PD. Many previous studies have described oscillations in similar, or higher than, tremor frequencies in the BG of MPTP monkeys (Bergman et al., 1994; Raz et al., 1996, 2000, 2001) and in human patients (Timmermann et al., 2003; Brown, 2006; Weinberger et al., 2006). However, the correlations between the BG oscillations and the tremor are transient and intermittent (Hurtado et al., 1999; Lemstra et al., 1999; Raz et al., 2000; Hurtado et al., 2005; Heimer et al., 2006). Based on the results of the present study, we suggest that the high-frequency 
oscillations in the $\mathrm{BG}$ do not directly drive the PD tremor. Rather, these BG oscillations should be considered as disrupting the normal motor processing of the MI (and motor brainstem centers) leading to the main core negative symptoms of $\mathrm{PD}$, akinesia and bradykinesia. As in many other cases of motor dysfunction and weakness (Elble and Koller, 1990), PD tremor emerges, perhaps as part of the compensatory mechanisms of the nervous system. According to this hypothesis, therapeutic DBS procedures may stop the tremor because they block the abnormal neural activity of the BG. Nevertheless, we are aware that artificial, nonspecific electrical stimulation at tremor frequencies may not adequately mimic the signals generated by tremor cells that comprise only a subset of the population of BG neurons. In addition, the filter properties in the BG network of human PD patients after many years of disease evolution and therapy may differ from those observed in the vervet MPTP model within only a few days after the degeneration of dopaminergic neurons (Langston et al., 1984; Di Paolo et al., 1986; Hara et al., 1987; Sundstrom et al., 1988).

Clinical studies define two extreme subtypes of PD: the akinetic-rigid and tremor-dominant (Jankovic et al., 1990). Similarly, MPTP-treated macaques develop only short episodes of high-frequency action/postural tremor (Burns et al., 1983) beyond akinesia and rigidity, whereas vervets commonly develop prolonged episodes of low-frequency tremor (Raz et al., 2000; Heimer et al., 2006). Previous studies of the pathological correlates of these clinical subtypes have failed to reach a consensus (Paulus and Jellinger, 1991; Hirsch et al., 1992). Our finding of low-pass filter properties of MI-muscle pathways, and the working hypothesis of a PD tremor generation downstream to the BG network, indicates that future research on the pathological correlates of the clinical subtypes of PD should be directed toward the brainstem and spinal cord motor systems.

\section{References}

Alberts WW (1972) A simple view of parkinsonian tremor, electrical stimulation of cortex adjacent to the rolandic fissure in awake man. Brain Res 44:357-369.

Albin RL, Young AB, Penney JB (1989) The functional anatomy of basal ganglia disorders. Trends Neurosci 12:366-375.

Alexander GE, DeLong MR (1985) Microstimulation of the primate neostriatum. I. Physiological properties of striatal microexcitable zones. J Neurophysiol 53:1401-1416.

Alexander GE, DeLong MR, Strick PL (1986) Parallel organization of functionally segregated circuits linking basal ganglia and cortex. Annu Rev Neurosci 9:357-381.

Asanuma H, Rosen I (1972) Topographical organization of cortical efferent zones projecting to distal forelimb muscles in the monkey. Exp Brain Res 14:243-256.

Baker SN, Olivier E, Lemon RN (1997) Coherent oscillations in monkey motor cortex and hand muscle EMG show task-dependent modulation. J Physiol (Lond) 501:225-241.

Bar-Gad I, Morris G, Bergman H (2003) Information processing, dimensionality reduction and reinforcement learning in the basal ganglia. Prog Neurobiol 71:439-473.

Bar-Gad I, Elias S, Vaadia E, Bergman H (2004) Complex locking rather than complete cessation of neuronal activity in the globus pallidus of a 1-methyl-4-phenyl-1,2,3,6-tetrahydropyridine-treated primate in response to pallidal microstimulation. J Neurosci 24:7410-7419.

Baratta RV, Solomonow M, Zhou BH (1998) Frequency domain-based models of skeletal muscle. J Electromyogr Kinesiol 8:79-91.

Ben Shaul Y, Stark E, Asher I, Drori R, Nadasdy Z, Abeles M (2003) Dynamical organization of directional tuning in the primate premotor and primary motor cortex. J Neurophysiol 89:1136-1142.

Benabid AL (2003) Deep brain stimulation for Parkinson's disease. Curr Opin Neurobiol 13:696-706.

Benabid AL, Deuschl G, Lang AE, Lyons KE, Rezai AR (2006) Deep brain stimulation for Parkinson's disease. Mov Disord 21 [Suppl 14]:S168-S170.
Benazzouz A, Boraud T, Dubedat P, Boireau A, Stutzmann JM, Gross C (1995) Riluzole prevents MPTP-induced parkinsonism in the rhesus monkey: a pilot study. Eur J Pharmacol 284:299-307.

Bergman H, Wichmann T, DeLong MR (1990) Reversal of experimental parkinsonism by lesions of the subthalamic nucleus. Science 249:1436-1438.

Bergman H, Wichmann T, Karmon B, DeLong MR (1994) The primate subthalamic nucleus. II. Neuronal activity in the MPTP model of Parkinsonism. J Neurophysiol 72:507-520.

Boraud T, Bezard E, Guehl D, Bioulac B, Gross C (1998) Effects of L-DOPA on neuronal activity of the globus pallidus externalis (GPe) and globus pallidus internalis (GPi) in the MPTP-treated monkey. Brain Res 787:157-160.

Brown P (2006) Bad oscillations in Parkinson's disease. J Neural Transm Suppl 27-30.

Burns RS, Chiueh CC, Markey SP, Ebert MH, Jacobowitz DM, Kopin IJ (1983) A primate model of parkinsonism: selective destruction of dopaminergic neurons in the pars compacta of the substantia nigra by $N$-methyl-4-phenyl-1,2,3,6-tetrahydropyridine. Proc Natl Acad Sci USA 80:4546-4550.

Chen CC, Litvak V, Gilbertson T, Kuhn A, Lu CS, Lee ST, Tsai CH, Tisch S, Limousin P, Hariz M, Brown P (2007) Excessive synchronization of basal ganglia neurons at $20 \mathrm{~Hz}$ slows movement in Parkinson's disease. Exp Neurol 205:214-221.

Cheney PD, Fetz EE (1985) Comparable patterns of muscle facilitation evoked by individual corticomotoneuronal (CM) cells and by single intracortical microstimuli in primates: evidence for functional groups of CM cells. J Neurophysiol 53:786-804.

Contreras CM, Mexicano G, Guzman-Flores CA (1981) Stereotaxic brain atlas of the green monkey (Cercopithecus aethiops aethiops). Bol Est Med Biol 31:383-428.

DeLong MR (1971) Activity of pallidal neurons during movement. J Neurophysiol 34:414-427.

DeLong MR (1990) Primate models of movement disorders of basal ganglia origin. Trends Neurosci 13:281-285.

Di Paolo T, Bedard P, Daigle M, Boucher R (1986) Long-term effects of MPTP on central and peripheral catecholamine and indoleamine concentrations in monkeys. Brain Res 379:286-293.

Doudet DJ, Gross C, Arluison M, Bioulac B (1990) Modifications of precentral cortex discharge and EMG activity in monkeys with MPTP-induced lesions of DA nigral neurons. Exp Brain Res 80:177-188.

Elble RJ, Koller WC (1990) Tremor. Baltimore: The Johns Hopkins UP.

Elias S, Joshua M, Goldberg JA, Heimer G, Arkadir D, Morris G, Bergman H (2007) Statistical properties of pauses of the high-frequency discharge neurons in the external segment of the globus pallidus. J Neurosci 27:2525-2538.

Evarts EV (1966) Pyramidal tract activity associated with a conditioned hand movement in the monkey. J Neurophysiol 29:1011-1027.

Filali M, Hutchison WD, Palter VN, Lozano AM, Dostrovsky JO (2004) Stimulation-induced inhibition of neuronal firing in human subthalamic nucleus. Exp Brain Res 156:274-281.

Filion M, Tremblay L, Bedard PJ (1988) Abnormal influences of passive limb movement on the activity of globus pallidus neurons in parkinsonian monkeys. Brain Res 444:165-176.

Freund HJ, Hefter H (1993) The role of basal ganglia in rhythmic movement. Adv Neurol 60:88-92.

Garcia L, D’Alessandro G, Bioulac B, Hammond C (2005) High-frequency stimulation in Parkinson's disease: more or less? Trends Neurosci 28:209-216.

Georgopoulos AP, Schwartz AB, Kettner RE (1986) Neuronal population coding of movement direction. Science 233:1416-1419.

Goldberg JA, Boraud T, Maraton S, Haber SN, Vaadia E, Bergman H (2002) Enhanced synchrony among primary motor cortex neurons in the 1-methyl-4-phenyl-1,2,3,6-tetrahydropyridine primate model of Parkinson's disease. J Neurosci 22:4639-4653.

Graziano MS, Taylor CS, Moore T (2002) Complex movements evoked by microstimulation of precentral cortex. Neuron 34:841-851.

Grosse P, Cassidy MJ, Brown P (2002) EEG-EMG, MEG-EMG and EMGEMG frequency analysis: physiological principles and clinical applications. Clin Neurophysiol 113:1523-1531.

Hammond C, Bergman H, Brown P (2007) Pathological synchronization in Parkinson's disease: networks, models and treatments. Trends Neurosci 30:357-364. 
Hara K, Tohyama I, Kimura H, Fukuda H, Nakamura S, Kameyama M (1987) Reversible serotoninergic neurotoxicity of $N$-methyl-4-phenyl1,2, 3,6-tetrahydropyridine (MPTP) in mouse striatum studied by neurochemical and immunohistochemical approaches. Brain Res 410:371-374.

Hashimoto T, Elder CM, Vitek JL (2002) A template subtraction method for stimulus artifact removal in high-frequency deep brain stimulation. J Neurosci Methods 113:181-186.

Hashimoto T, Elder CM, Okun MS, Patrick SK, Vitek JL (2003) Stimulation of the subthalamic nucleus changes the firing pattern of pallidal neurons. J Neurosci 23:1916-1923.

Heimer G, Bar-Gad I, Goldberg JA, Bergman H (2002) Dopamine replacement therapy reverses abnormal synchronization of pallidal neurons in the 1-methyl-4-phenyl-1,2,3,6-tetrahydropyridine primate model of Parkinsonism. J Neurosci 22:7850-7855.

Heimer G, Rivlin-Etzion M, Bar-Gad I, Goldberg JA, Haber SN, Bergman H (2006) Dopamine replacement therapy does not restore the full spectrum of normal pallidal activity in the 1-methyl-4-phenyl-1,2,3,6-tetrahydropyridine primate model of Parkinsonism. J Neurosci 26:8101-8114.

Hirsch EC, Mouatt A, Faucheux B, Bonnet AM, Javoy Agid F, Graybiel AM, Agid Y (1992) Dopamine, tremor, and Parkinson's disease [letter]. Lancet 340:125-126.

Horak FB, Anderson ME (1984) Influence of globus pallidus on arm movements in monkeys. II. Effects of stimulation. J Neurophysiol 52:305-322.

Hurtado JM, Gray CM, Tamas LB, Sigvardt KA (1999) Dynamics of tremorrelated oscillations in the human globus pallidus: a single case study. Proc Natl Acad Sci USA 96:1674-1679.

Hurtado JM, Rubchinsky LL, Sigvardt KA, Wheelock VL, Pappas CT (2005) Temporal evolution of oscillations and synchrony in GPi/muscle pairs in Parkinson's disease. J Neurophysiol 93:1569-1584.

Jankovic J, McDermott M, Carter J, Gauthier S, Goetz C, Golbe L, Huber S, Koller W, Olanow C, Shoulson I (1990) Variable expression of Parkinson's disease: a base-line analysis of the DATATOP cohort. The Parkinson Study Group. Neurology 40:1529-1534.

Joel D, Weiner I (1994) The organization of the basal gangliathalamocortical circuits: open interconnected rather than closed segregated. Neuroscience 63:363-379.

Joshua M, Elias S, Levine O, Bergman H (2007) Quantifying the isolation quality of extracellularly recorded action potentials. J Neurosci Methods 163:267-282.

Kilner JM, Baker SN, Salenius S, Jousmaki V, Hari R, Lemon RN (1999) Task-dependent modulation of $15-30 \mathrm{~Hz}$ coherence between rectified EMGs from human hand and forearm muscles. J Physiol (Lond) 516:559-570.

Kita H (1992) Responses of globus pallidus neurons to cortical stimulation: intracellular study in the rat. Brain Res 589:84-90.

Langston JW, Irwin I, Langston EB (1984) A comparison of the acute and chronic effects of 1-methyl-4-phenyl-1,2,5,6-tetrahydropyridine (MPTP)-induced parkinsonism in humans and the squirrel monkey. Neurology 34 [Suppl 1]:268.

Lathi BP (2005) Linear systems and signals. New York: Oxford UP.

Leblois A, Boraud T, Meissner W, Bergman H, Hansel D (2006) Competition between feedback loops underlies normal and pathological dynamics in the basal ganglia. J Neurosci 26:3567-3583.

Lemstra AW, Verhagen ML, Lee JI, Dougherty PM, Lenz FA (1999) Tremor-frequency $(3-6 \mathrm{~Hz})$ activity in the sensorimotor arm representation of the internal segment of the globus pallidus in patients with Parkinson's disease. Neurosci Lett 267:129-132.

Maltete D, Jodoin N, Karachi C, Houeto JL, Navarro S, Cornu P, Agid Y, Welter ML (2007) Subthalamic stimulation and neuronal activity in the substantia nigra in Parkinson's disease. J Neurophysiol 97:4017-4022.

Martin RF, Bowden DM (2000) Primate brain maps: structure of the macaque brain. Amsterdam: Elsevier Science.

Maurice N, Deniau JM, Glowinski J, Thierry AM (1999) Relationships between the prefrontal cortex and the basal ganglia in the rat: physiology of the cortico-nigral circuits. J Neurosci 19:4674-4681.

McHaffie JG, Stanford TR, Stein BE, Coizet V, Redgrave P (2005) Subcortical loops through the basal ganglia. Trends Neurosci 28:401-407.

McIntyre CC, Grill WM (2002) Extracellular stimulation of central neurons: influence of stimulus waveform and frequency on neuronal output. J Neurophysiol 88:1592-1604.
Mena-Segovia J, Bolam JP, Magill PJ (2004) Pedunculopontine nucleus and basal ganglia: distant relatives or part of the same family? Trends Neurosci 27:585-588.

Nambu A, Tokuno H, Hamada I, Kita H, Imanishi M, Akazawa T, Ikeuchi Y, Hasegawa N (2000) Excitatory cortical inputs to pallidal neurons via the subthalamic nucleus in the monkey. J Neurophysiol 84:289-300.

Nini A, Feingold A, Slovin H, Bergman H (1995) Neurons in the globus pallidus do not show correlated activity in the normal monkey, but phaselocked oscillations appear in the MPTP model of Parkinsonism. J Neurophysiol 74:1800-1805.

Palmer SS, Fetz EE (1985) Effects of single intracortical microstimuli in motor cortex on activity of identified forearm motor units in behaving monkeys. J Neurophysiol 54:1194-1212.

Paulus W, Jellinger K (1991) The neuropathologic basis of different clinical subgroups of Parkinson's disease. J Neuropathol Exp Neurol 50:743-755.

Ranck JB (1975) Which elements are excited in electrical stimulation of mammalian central nervous system: a review. Brain Res 98:417-440.

Rav-Acha M, Sagiv N, Segev I, Bergman H, Yarom Y (2005) Dynamic and spatial features of the inhibitory pallidal GABAergic synapses. Neuroscience 135:791-802.

Raz A, Feingold A, Zelanskaya V, Vaadia E, Bergman H (1996) Neuronal synchronization of tonically active neurons in the striatum of normal and parkinsonian primates. J Neurophysiol 76:2083-2088.

Raz A, Vaadia E, Bergman H (2000) Firing patterns and correlations of spontaneous discharge of pallidal neurons in the normal and the tremulous 1-methyl-4-phenyl-1,2,3,6-tetrahydropyridine vervet model of Parkinsonism. J Neurosci 20:8559-8571.

Raz A, Frechter-Mazar V, Feingold A, Abeles M, Vaadia E, Bergman H (2001) Activity of pallidal and striatal tonically active neurons is correlated in mptp-treated monkeys but not in normal monkeys. J Neurosci 21:RC128(1-5).

Rivlin-Etzion M, Marmor O, Heimer G, Raz A, Nini A, Bergman H (2006) Basal ganglia oscillations and pathophysiology of movement disorders. Curr Opin Neurobiol 16:629-637.

Ryan LJ, Clark KB (1991) The role of the subthalamic nucleus in the response of globus pallidus neurons to stimulation of the prelimbic and agranular frontal cortices in rats. Exp Brain Res 86:641-651.

Salenius S, Hari R (2003) Synchronous cortical oscillatory activity during motor action. Curr Opin Neurobiol 13:678-684.

Shink E, Sidibe M, Smith Y (1997) Efferent connections of the internal globus pallidus in the squirrel monkey. II. Topography and synaptic organization of pallidal efferents to the pedunculopontine nucleus. J Comp Neurol 382:348-363.

Song DD, Haber SN (2000) Striatal responses to partial dopaminergic lesion: evidence for compensatory sprouting. J Neurosci 20:5102-5114.

Strafella AP, Ko JH, Grant J, Fraraccio M, Monchi O (2005) Corticostriatal functional interactions in Parkinson's disease: a rTMS/[11C]raclopride PET study. Eur J Neurosci 22:2946-2952.

Sundstrom E, Luthman J, Goldstein M, Jonsson G (1988) Time course of MPTP-induced degeneration of the nigrostriatal dopamine system in C57BL/6 mice. Brain Res Bull 21:257-263.

Timmermann L, Gross J, Dirks M, Volkmann J, Freund HJ, Schnitzler A (2003) The cerebral oscillatory network of parkinsonian resting tremor. Brain 126:199-212.

Topka H, Mescheriakov S, Boose A, Kuntz R, Hertrich I, Seydel L, Dichgans J, Rothwell J (1999) A cerebellar-like terminal and postural tremor induced in normal man by transcranial magnetic stimulation. Brain 122:1551-1562.

Tremblay L, Filion M, Bedard PJ (1989) Responses of pallidal neurons to striatal stimulation in monkeys with MPTP-induced parkinsonism. Brain Res 498:17-33.

Weinberger M, Mahant N, Hutchison WD, Lozano AM, Moro E, Hodaie M, Lang AE, Dostrovsky JO (2006) Beta oscillatory activity in the subthalamic nucleus and its relation to dopaminergic response in Parkinson's disease. J Neurophysiol 96:3248-3256.

Wichmann T (2000) A digital averaging method for removal of stimulus artifacts in neurophysiologic experiments. J Neurosci Methods 98:57-62.

Wu YR, Levy R, Ashby P, Tasker RR, Dostrovsky JO (2001) Does stimulation of the GPi control dyskinesia by activating inhibitory axons? Mov Disord 16:208-216. 